\title{
Dynamics of counterpropagating waves in parametrically forced systems
}

\author{
Carlos Martel ，Edgar Knobloch ， José M. Vega \\ E.T.S.I. Aeronáuticos. Universidad Politécnica de Madrid. 28040 Madrid. Spain \\ Department of Physics. Liniversing of California at Berkeley. Berkeley. CA 94720. LSA
}

\begin{abstract}
Parametrically driven waves in weakly dissipative systems with one extended dimension are considered. Multiple scale techniques are used to derive amplitude equations describing the interaction between counterpropagating waves. Dissipation. detuning and forcing are all assumed to be weak and any coupling to mean fields (such as large scale flows in fluid systems) is ignored. If the aspect ratio is moderately large the system is described by a pair of nonlocal equations for the (complex) amplitudes of the waves. The dynamics of these equations are studied both in annular and bounded geometries with lateral walls. The equations admit spatially uniform solutions in the form of standing waves and spatially nonuniform solutions with both simple and complex time-dependence. 'lransitions among these states are investigated as a function of the driving in three particular cases. 02000 Elsevier Science B.V. All rights reserved.
\end{abstract}

PACS: $47.20 . \mathrm{Ky} ; 47.54 .+\mathrm{r}$

Keywords: Parametrically excited waves: Amplitude equations; Counterpropagating waves: Multiple scale techniques

\section{Introduction}

In the last few years there has been considerable interest in the dynamics of counterpropagating waves in parametrically driven systems. The simplest situation arises when only one unbounded direction, hereafter referred to as the $x$ direction, is present. In such systems waves can propagate to the left or right but, as shown by Riecke et al. [1] and Walgraef [2], the parametric forcing provides a coupling which favors, at small amplitude, the presence of an equal amplitude superposition of these waves, hereafter referred to as standing waves (SW). Typical examples arise in the Faraday system on a line (or in annular geometry) in which surface gravity-capillary waves are excited by vertical acceleration of the container with frequency $2 \omega$; the frequency of the resulting waves is then near $\omega$, i.e., near half of the driving frequency [3]. A similar situation arises in parametrically excited edge waves [4]. These waves are 
longshore waves driven by waves normally incident on a sloping beach. Related situations also arise in nonlinear optics [5] and more generally in systems undergoing an oscillatory instability in the presence of parametric forcing $[6,7]$.

In the present paper we are interested in weakly dissipative systems with one extended dimension. We consider systems that are invariant under spatial translations and reflection in this direction and assume that in the absence of parametric forcing all waves decay. Near threshold we write the physical variables in the form

$$
\mathbf{u}(x, t)=\left\{A(x, t) \mathrm{e}^{\mathrm{i} \omega t+\mathrm{i} k x}+B(x, t) \mathrm{e}^{\mathrm{i} \omega t-\mathrm{i} k x}+\text { c.c. }\right\} \mathbf{u}_{0}+\cdots,
$$

where $\omega$ is half the driving frequency and $k \equiv k(\omega)$ is the corresponding wave number, and focus on patterns with (large) spatial period $L$. We assume that the wave amplitudes $A$ and $B$ are small and that $\left|A_{x x}\right| \ll\left|A_{x}\right| \ll$ $|A|,\left|B_{x x}\right| \ll\left|B_{x}\right| \ll|B|,\left|A_{l}\right| \ll|\Lambda|,\left|B_{l}\right| \ll|B|$, i.e., that they also vary slowly in both space and time. A standard multiple scale procedure then leads to a pair of equations of the form, cf. $[8]$

$$
\begin{aligned}
& A_{t}=\mathrm{i} \alpha A_{x x}+c_{g} A_{x}-\delta A+\mathrm{i}\left(\beta|A|^{2}+\gamma|B|^{2}\right) A+\mu \bar{B}, \\
& B_{l}=\mathrm{i} \alpha B_{x x}-c_{g} B_{x}-\delta B+\mathrm{i}\left(\beta|B|^{2}+\gamma|A|^{2}\right) B+\mu \bar{A} .
\end{aligned}
$$

Here $\mu>0$ denotes the (small) amplitude of the parametric forcing; the coefficients $\delta>0, \alpha$ and $c_{g}$ measure the decay rate, dispersion and group velocity of the wavetrains in the absence of forcing. The coefficients of the nonlinear self- and cross-interaction terms are denoted by $\beta$ and $\gamma$, and are assumed to be such that $\beta+\gamma \neq 0$.

In an annular domain of length $L$ the spatial periodicity implies that the boundary conditions for $A$ and $B$ are

$$
A(x+L, t) \mathrm{e}^{\mathrm{i} \theta}=A(x, t), \quad B(x+L, t) \mathrm{e}^{-\mathrm{i} \theta}=B(x, t),
$$

where $\theta=k L(\bmod 2 \pi)$ measures the mismatch between the natural wavelength of the pattern and the domain period.

The change of variables $\tilde{A}=A e^{\mathrm{i} \theta x / L}, \tilde{B}=B e^{-\mathrm{i} \theta x / L}$, corresponding to changing the wave number from $k$ to $\tilde{k}=k-\theta / L$ (the closest wave number allowed by the periodicity), transforms Eqs. (2)-(4) into the form

$$
\begin{aligned}
& A_{t}=\mathrm{i} \alpha A_{x x}+A_{x}-(\delta+\mathrm{i} \nu) A+\mathrm{i}\left(\beta|A|^{2}+\gamma|B|^{2}\right) A+\mu \bar{B}, \\
& B_{t}=\mathrm{i} \alpha B_{x x}-B_{x}-(\delta+\mathrm{i} \nu) B+\mathrm{i}\left(\beta|B|^{2}+\gamma|A|^{2}\right) B+\mu \bar{A}, \\
& A(x+L, t)=\Lambda(x, t), \quad B(x+L, t)=B(x, t) .
\end{aligned}
$$

In writing these equations we have dropped the tildes and introduced the quantity $v \equiv \omega-\Omega$ that takes into account the small detuning between $\omega$ and the closest natural frequency of the system, $\Omega$, associated with the shifted wave number. Thus the detuning is nonzero when the wavelength selected by the driving frequency is incommensurate with the prescribed spatial period. In addition we have chosen the unit of time such that the group speed of the waves with the shifted wave number is one, assuming that the phase and group velocities of the waves have the same sign; if this is not the case the roles of $A$ and $B$ are interchanged. The shift in the wave number also introduces small corrections to $\alpha, \delta$ and the coefficients $\beta, \gamma$ of the nonlinear terms which we absorb into the definition of these coefficients.

The solutions of Eqs. (5)-(7) depend on the domain size $L$ and the magnitude of the damping. detuning and forcing. There are two regimes that are of particular interest. To identify these we note that the stability of the trivial state $A=B=0$ with respect to perturbations with (complex) growth rate $s$ and wave number $2 \pi n / L$ is described by the dispersion relation:

$$
(s+8)^{2}+\left[v-2 \pi n / L+\alpha(2 \pi n / L)^{2}\right]^{2}-\mu^{2}=0 .
$$



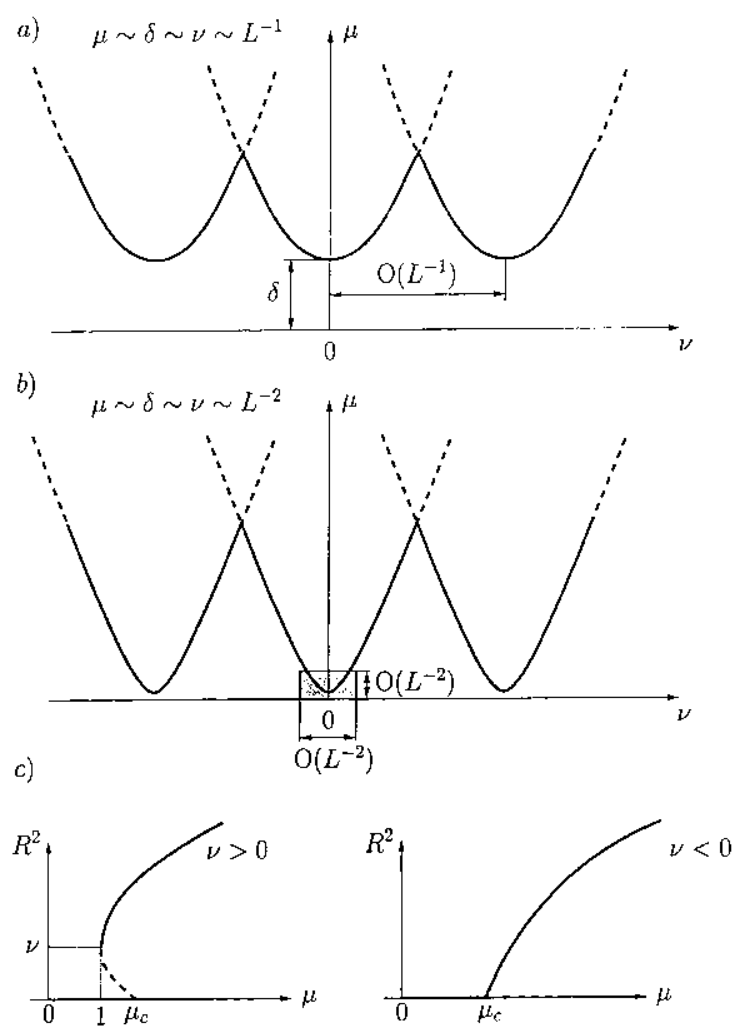

Fig. 1. Instability threshold (solid line) for the trivial state $A=B=0$ from (a) Fqs. (5)-(7) and (b) (shaded region) Fiqs. (38)-(40). (c) Sub and super-critical branches of standing waves from (38)-(40) for $v>0$ and $v<0$. The parameters $\ell \ell$ and $v$ are unscaled in (a.b) as in Eqs. (5) and (6). but are scalcd in (c) as in Eqs. (38) and (39).

Thus for each integer $n$ there is a tongue of instability $\mu>\mu_{n}$,

$$
\mu_{n}^{2} \equiv \delta^{2}+\left[v-2 \pi n / L+\alpha(2 \pi n / L)^{2}\right]^{2},
$$

whose minimum is independent of both $n$ and the detuning $v$ (see Fig. 1a). The instability gives rise to nontrivial solutions in the form of uniform standing waves (SW), $|A|=|B|=$ constant; these may in turn be unstable with respect to wavelength-changing side-band instabilities as in the experiments [9]. However, as suggested by Fig. la, if $\delta \ll 1$ and $L \gg 1$ these wavelength-changing instabilities can be described by Eqs. (5)-(7) only if $\mathcal{O}\left(L^{-1}\right)$ variation of $v$ is allowed; if the variation of $v$ about a tongue minimum is restricted to be $\mathcal{O}\left(L^{-2}\right)$, and both damping and forcing remain sufficiently small (see Fig. $1 \mathrm{~b}$ ), wavelength-changing instabilities cannot occur. For the $n=0$ tongue this is the case in the distinguished limit:

$$
\delta \sim v \sim \mu \sim L^{-2} .
$$

In this limit the advection at the group velocity is fast relative to the growth rate of the instability and we write $(A, B)=L^{-1}(\tilde{A}, \tilde{B}),(x, t)=L(\tilde{x}, \tilde{f})$ and suppose that $(\delta, v, \mu)=L^{-2}(\tilde{\delta}, \tilde{v}, \tilde{\mu})$. Dropping tildes, we obtain

$$
\begin{aligned}
& A_{t}-A_{x}=L^{-1}\left(\mathrm{i} \alpha A_{x x}-(\delta+\mathrm{i} \nu) A+\mathrm{i}\left(\beta|A|^{2}+\gamma|B|^{2}\right) A+\mu \bar{B}\right), \\
& B_{t}+B_{x}=L^{-1}\left(\mathrm{i} \alpha B_{x x}-(\delta+\mathrm{i} \nu) B+\mathrm{i}\left(\beta|B|^{2}+\gamma|A|^{2}\right) B+\mu \bar{A}\right) .
\end{aligned}
$$


It follows that $A=A_{0}(\eta, \tau)+L^{-1} A_{1}(\eta, \xi, \tau)+\cdots, B=B_{0}(\xi, \tau)+L^{-1} B_{1}(\eta, \xi, \tau)+\cdots$, where $\eta=x+l$, $\xi=x-l$ and $\tau=l / L^{2}$. At $\mathcal{O}\left(L^{-1}\right)$ one now obtains

$$
\begin{aligned}
& -2 A_{1 \xi}=-A_{0 \tau}+\mathrm{i} \alpha A_{0 \eta \eta}-(\delta+\mathrm{i} v) A_{0}+\mathrm{i}\left(\beta\left|A_{0}\right|^{2}+\gamma\left|B_{0}\right|^{2}\right) A_{0}+\mu \bar{B}_{0}, \\
& +2 B_{1 \eta}=-B_{0 \tau}+\mathrm{i} \alpha B_{0 \xi \xi}-(\delta+\mathrm{i} v) B_{0}+\mathrm{i}\left(\beta\left|B_{0}\right|^{2}+\gamma\left|A_{0}\right|^{2}\right) B_{0}+\mu \bar{A}_{0} .
\end{aligned}
$$

The solvability conditions for these two equations now give

$$
\begin{aligned}
& A_{0 \tau}=\mathrm{i} \alpha A_{0 m}-(\delta+\mathrm{i} v) A_{0}+\mathrm{i}\left(\beta\left|A_{0}\right|^{2}+\gamma\left\langle\left|B_{0}\right|^{2}\right\rangle\right) A_{0}+\mu\left\langle\bar{B}_{0}\right\rangle, \\
& B_{0 \tau}=\mathrm{i} \alpha B_{0 \xi \xi}-(\delta+\mathrm{i} v) B_{0}+\mathrm{i}\left(\beta\left|B_{0}\right|^{2}+\gamma\left\langle\left|A_{0}\right|^{2}\right\rangle\right) B_{0}+\mu\left\langle\bar{A}_{0}\right\rangle,
\end{aligned}
$$

with

$$
A_{0}(\eta+1, \tau)=A_{0}(\eta, \tau), \quad B_{0}(\xi+1, \tau)=B_{0}(\xi, \tau)
$$

Here $\langle\ldots\rangle \equiv \int_{0}^{1} \ldots \mathrm{d} \eta$ or $\int_{0}^{1} \ldots \mathrm{d} \xi$ denotes an average over the spatial variable $\eta$ or $\xi$.

Eqs. (15) and (16) describe the slow dynamics at sufficiently small forcing, damping and detuning for fixed $L \gg 1$, or equivalently, for large (but not too large) values of $L$ and fixed, small values of $\mu, \delta$ and $\nu$. In the following we refer to these as the nonlocal equations, and drop the subscript 0 on the amplitudes $A_{0}, B_{0}$. For strongly dissipative systems related nonlocal equations are obtained in $[10-12]$.

The second regime of interest applies when $\delta \sim v \sim \mu=\mathcal{O}\left(L^{-1}\right)$. In this case, if $\partial_{x}$ and $\partial_{t}$ are both $\mathcal{O}\left(L^{-1}\right)$ the dispersion terms are of higher order $\left(\mathcal{O}\left(L^{-2}\right)\right)$ and Eqs. (5) and $(6)$ are replaced by the hyperbolic (and local) system

$$
\begin{aligned}
& A_{t}-\Lambda_{x}=-(\delta+\mathrm{i} v) \Lambda+\mathrm{i}\left(\beta|\Lambda|^{2}+\gamma|B|^{2}\right) A+\mu \bar{B}, \\
& B_{t}+B_{x}=-(\delta+\mathrm{i} v) B+\mathrm{i}\left(\beta|B|^{2}+\gamma|\Lambda|^{2}\right) B+\mu \bar{A},
\end{aligned}
$$

cf. [12-14]. As will be discussed in detail elsewhere, equations of this type need not be well posed. This is because in some circumstances the integration generates small scale motions. When these scales are $\mathcal{O}\left(L^{-1}\right)$ the replacement of Eqs. (5) and (6) by Eqs. (18) and (19) is invalidated, i.e., the appearance of scales of this size in the integration indicates a breakdown of the hy perbolic system. Moreover, even if these equations are well-posed, they can provide a poor approximation to (5) and (6) because of the neglected higher order dispersive terms, cf. $[12,14]$. On the other hand, in the present case the parametric forcing couples the phases of $A$ and $B$ and thereby introduces a new source of complex dy namics into the system (18) and (19) that is absent in the unforced case studied by Martel and Vega $[12,14]$. In particular, for reasons already mentioned, the forced hyperbolic system (18) and (19) does admit side-band instabilities.

In the present paper we focus on the dy namics described by the nonlocal equations (15)-(17) only. These equations have four symmetries,

$$
\eta \rightarrow \eta+\text { constant }, \quad \eta \rightarrow-\eta, \quad \xi \rightarrow \xi+\text { constant, } \quad \xi \rightarrow-\xi,
$$

in addition to

$$
\hat{\sigma}:(A, B) \rightarrow\left(A \mathrm{e}^{\mathrm{i} /}, B \mathrm{e}^{-\mathrm{i} \sigma}\right), \quad \hat{\kappa}:(A, B) \rightarrow(B, A) .
$$

The former is the manifestation of Iranslation invariance on fast spatial scales; the latter will be referred to as the interchange symmetry. However, only the translation symmetry $x \rightarrow x+$ constant and the symmetries $\hat{\sigma}$ and

$$
A \leftrightarrow B, \quad x \leftrightarrow-x,
$$


are symmetries of Eqs. (5)-(7); the symmetry (22) is a consequence of the invariance of the original physical problem under spatial reflection. Thus a solution of (15)-(17) yields a reflection-symmetric solution of (5)-(7) if and only if it is imariant under the symmetry

$$
\Lambda \leftrightarrow B \mathrm{e}^{\mathrm{i} \sigma_{0}}, \quad \xi \leftrightarrow-\eta+c_{0},
$$

for some constants $\sigma_{0}$ and $c_{0}$. The additional symmetrics are symmetrics of the leading order averaged cquations only, but are not expected to be present at higher order (cᄃ. [15]). Thus care must be taken not to overinterpret the sy mmetries of the solutions of the leading order equations (15)-(17).

In Section 2, we describe the modifications to the averaged equations that are necessary in order to describe the corresponding dynamics in a system with lateral walls. Our weakly dissipative formulation requires that we take the reflection coefficient at the walls to be near unity, i.c., that the sidewalls be almost perfectly reflecting. We then summarize in Section 3 the linear stability properties of the trivial state and of nontrivial but spatially uniform solutions of both sets of equations. Section 4 describes the properties of these equations in the four distinct cases identified by the linear stability analysis in Section 3. Throughout we take $\mu$ to be the bifurcation parameter and study numerically the sequence of transitions as $\mu$ increases. We find a large variety of different solution types including states we call standing waves in which $A=B$ with both uniform in space, states in which $A=B$ but both are nonuniform, states in which $A \neq B$ with one amplitude (e.g., $A$ ) uniform in space and the other $(B)$ nonuniform, and finally states in which both are nonuniform and different. Each of these states can exhibit different types of dynamics in time. Because of (20) and (21) these conditions are defined modulo translations, reflection and phase shifts. It should be noted that since $A(\eta, \tau) \equiv A(x+\ell, \tau)$ and $B(\xi, \tau) \equiv B(x-\ell, \tau)$ a state in which $A=B$ but $A$ is nonuniform actually corresponds to an oscillatory state with (periodic) time-dependence on the advection timescale $t$. Any time-dependence arising from the slower timescale $\tau$ is superposed on top of this faster oscillation. Aside from numerous instances of hysteresis and chaos associated with the transitions between the above states we also describe states that could be interpreted as describing modes reminiscent of those seen in experiments on the Faraday system [9]. However, the preliminary treatment discussed in the present paper is not a rigorous description of the Faraday system because of the neglect of large scale hydrodynamic flows. This issue is addressed in Section 5 and will form the focus of a subsequent paper.

\section{Effect of distant side boundaries}

We consider next the situation in which the spatial domain is bounded laterally by two boundaries a distance $L \gg 1$ apart. Then the amplitude equations (2) and (3) apply only in the domain $-L / 2<x<L / 2$ and the appropriate boundary conditions are

$$
\begin{array}{lll}
B=r A, & c_{g}\left(B_{x}+r A_{x}\right)=\left(1-|r|^{2}\right)\left\lfloor\mu \bar{A}+\mathrm{i}(\gamma-\beta) r|A|^{2} A\right] & \text { at } x=-L / 2, \\
A=r B, & c_{g}\left(A_{x}+r B_{x}\right)=\left(|r|^{2}-1\right)\left[\mu \bar{B}+\mathrm{i}(\gamma-\beta) r|B|^{2} B\right] & \text { at } x=L / 2,
\end{array}
$$

where $r \neq 0$ is a complex reflection coefficient assumed to be identical for both boundaries. These boundary conditions are obtained by matching the solution in the bulk $(-L / 2<x<L / 2)$ to the boundary layers in the regions $|x \pm L / 2| \sim 1$ as described by Martel and Vega [12]. Note that these boundary layers are wide, but under our assumptions do not extend across the whole domain. The distinguished limit discussed here is

$$
0<\delta-\frac{\ln |r|}{L} \sim \delta \sim\left|\frac{\text { phase } r}{L}\right| \sim \mu \sim|A|^{2} \sim|B|^{2} \sim L^{-2},
$$


and corresponds to almost perfectly reflecting sidewalls: $|r-1| \sim L^{-1}$. Proceeding as in the previous section, we introduce the new variables (and parameters)

$$
\begin{aligned}
& \tilde{A}=L r^{-x / t} A / \sqrt{c_{g}}, \quad \tilde{B}=L r^{x / L} B / \sqrt{c_{g}}, \quad \tilde{x}=x / L, \quad \tilde{\imath}=l c_{g} / L, \\
& \tilde{\delta}=\left(\delta-\frac{\ln |r|}{L}\right) \frac{L^{2}}{c_{g}}, \quad \tilde{v}=\left(-\frac{\text { phase } r}{L}\right) \frac{L^{2}}{c_{g}}, \quad \tilde{\mu}=\mu L^{2} / c_{g}, \quad \tilde{\alpha}=\alpha / c_{g},
\end{aligned}
$$

allowing us to rewrite (2) and (3), and (24) and (25) in the form (after dropping the tildes)

$$
\begin{aligned}
& A_{l}-\Lambda_{x}=L^{-1}\left[\mathrm{i} \alpha \Lambda_{x x}-(\delta+\mathrm{i} \nu) A+\mathrm{i}\left(\beta|A|^{2}+\gamma|B|^{2}\right) \Lambda+\mu \bar{B}\right\rceil+\mathcal{O}\left(L^{-2}\right), \\
& B_{l}+B_{x}=L^{-1}\left[\mathrm{i} \alpha B_{x x}-(\delta+\mathrm{i} \nu) B+\mathrm{i}\left(\beta|B|^{2}+\gamma|\Lambda|^{2}\right) B+\mu \bar{\Lambda}\right\rceil+\mathcal{O}\left(L^{-2}\right),
\end{aligned}
$$

with

$$
A=B, \quad A_{x}+B_{x}=\mathcal{O}\left(L^{-2}\right) \quad \text { at } x= \pm \frac{1}{2} .
$$

Here $\delta$ and $v$ are the effective decay rate and detuning in the presence of the boundaries; the sidewalls amplify or damp the waves depending on whether $|r|>1$ or $|r|<1$. In writing these equations we have again assumed that the group and phase velocities are of the same sign. If this is not the case the effective reflection coefficient becomes $r^{-1}$, as discussed in [12] in the context of an oscillatory instability in large but bounded domains. Thus in the new variables the problem has been transformed into one with perfectly reflecting boundary conditions and if we neglect the $\mathcal{O}\left(L^{-2}\right)$ terms (as we hereafter do) we can relate the resulting solutions to those of the problem with periodic boundary conditions. We do this by defining the new variable $C$ by

$$
\begin{aligned}
& C(x, l)=A(x, l) \text { in }-\frac{1}{2} \leq x \leq \frac{1}{2}, \quad C(x, l)=B(1-x, l) \text { in } \frac{1}{2} \leq x \leq \frac{3}{2} \\
& C(x+2, l)=C(x, l)
\end{aligned}
$$

for all $x$. Thus $C$ has continuous second derivatives on the entire line and satisfies

$$
C_{t}(x, l)-C_{x}(x, l)=L^{-1}\left[\mathrm{i} \alpha C_{x x}(x, l)-(\delta+\mathrm{i} v) C(x, l)+\mathrm{I}\right],
$$

where

$$
\mathrm{I}=\mathrm{i}\left(\beta|C(x, t)|^{2}+\gamma|C(1-x, t)|^{2}\right) C(x, t)+\mu \bar{C}(1-x, t) .
$$

Then, if we proceed as in Section 1 when deriving Eqs. (15) and (16), we readily conclude that, to leading order,

$$
\begin{aligned}
& C_{0 \tau}=\mathrm{i} \alpha C_{0 \eta \eta}-(\delta+\mathrm{i} v) C_{0}+\mathrm{i}\left(\beta\left|C_{0}\right|^{2}+\gamma\left\langle\left|C_{0}\right|^{2}\right\rangle\right) C_{0}+\mu\left\langle\bar{C}_{0}\right\rangle, \\
& C_{0}(\eta+1, \tau)=C_{0}(\eta, \tau), \quad \text { for all } \eta .
\end{aligned}
$$

In writing Eqs. (36) and (37) we have rescaled the characteristic variable $\eta$ so as to make the spatial period cqual to 1. This cntails a rescaling of $\alpha$ to its correct value for a domain of length 1 . Again, (...) denotes a spatial average with respect to $\eta$. In the following we drop the subscript on $C_{0}$.

As in the annular geometry the physical interpretation of the solutions of this equation requires going back to the definition (32) and (33). Thus a state in which $C$ is nonuniform in $\eta$ corresponds to a state with oscillations on the fast advection timescale; any time-dependence on the slower timescale $\tau$ is superposed on such oscillations.

The appearance of the periodic boundary conditions (37) should come as no surprise, since problems with Neumann boundary conditions can always be cmbedded in problems with periodic boundary conditions with period 
twice the domain length [16]. Because of this construction the resulting problem (36) and (37) is equivariant under both translations $\eta \rightarrow \eta+$ constant, and reflections $\eta \rightarrow-\eta$, i.e., the presence of these symmetries is a consequence of the Neumann boundary conditions required by our scaling. Moreover, Eqs. (36) and (37) are a restriction of Eqs. (15)-(17) to the invariant subspace $A=B$, up to a trivial constant phase shift. This subspace includes the primary solution branch. However, not all global attractors of (15)-(17) are of this type and hence in some cases Eqs. (36) and (37) exhibit new dynamics. We discuss the differences between the solutions of Eqs. (15)-(17) and (36) and (37) in Section 4.

\section{Basic properties of the equations}

In this section we describe some basic propertics of the nonlocal problems (15)-(17) and (36). After rescaling $\tau$ and $(A, B)$ (and taking the complex conjugate in (15)-(17) and (36) and (37) ir $\beta+\gamma<0$ ) we can sel $\delta=1$ and $\beta+\gamma=1$. Under these conditions the two problems take the form

$$
\begin{aligned}
& \left.A_{\tau}=\mathrm{i} \alpha A_{\eta \eta}-(1+\mathrm{i} v) A+\left.\mathrm{i}|\beta| A\right|^{2}+(1-\beta)\left\langle|B|^{2}\right\rangle\right] A+\mu\langle\bar{B}\rangle, \\
& B_{\tau}=\mathrm{i} \alpha B_{\xi \xi}-(1+\mathrm{i} v) B+\mathrm{i}\left[\beta|B|^{2}+(1-\beta)\left\langle|A|^{2}\right\rangle\right] B+\mu\langle\bar{A}\rangle, \\
& A(\eta+1, \tau)=\Lambda(\eta, \tau), \quad B(\xi+1, \tau)=B(\xi, \tau),
\end{aligned}
$$

and

$$
\begin{aligned}
& C_{\tau}=\mathrm{i} \alpha C_{\eta \eta}-(1+\mathrm{i} \nu) C+\left.\mathrm{i}|\beta| C\right|^{2}+(1-\beta)\left\langle|C|^{2}\right\rangle \mid C+\mu\langle\bar{C}\rangle \\
& C(\eta+1, \tau)=C(\eta, \tau) .
\end{aligned}
$$

\subsection{Linear theory}

The linear stability properties of the trivial solution $A=B=0$ within Eqs. (38)-(40) are casily described. Infinitesimal perturbations of the form $\operatorname{cxp}(s \tau)$ with wave number $2 \pi n$ satisfy the dispersion relation

$$
(s+1)^{2}+v^{2}=\mu^{2} \quad \text { if } n=0, \quad(s+1)^{2}+v_{n}^{2}=0 \quad \text { if } n \neq 0,
$$

where

$$
v_{n}=v+\alpha(2 \pi n)^{2} .
$$

Thus instability sets in at $\mu_{c}=\sqrt{1+v^{2}}$, with $n=0$, in agrecment with Eq. (9) in the limit (10). For $n \neq 0$, Res $=$ -1 and hence all nonuniform modes decay. Morcover, the exact relation

$$
\frac{\mathrm{d}}{\mathrm{d} \tau}\left(\int_{0}^{1}|A|^{2} \mathrm{~d} \xi-\int_{0}^{1}|B|^{2} \mathrm{~d} \eta\right)=-2\left(\int_{0}^{1}|A|^{2} \mathrm{~d} \xi-\int_{0}^{1}|B|^{2} \mathrm{~d} \eta\right)
$$

provides a first integral and indicates that the manifold $\left\langle|A|^{2}\right\rangle=\left\langle|B|^{2}\right\rangle$ is invariant and globally attracting. Consequently, spatially uni form progressive waves (i.c., solutions of the type $|A|=$ constant, $|B|=$ constant, $|A| \neq|B|$ ) do not appear. This is in contrast to the related problem of a parametrically forced Hop $\Gamma$ bifurcation that is described by similar equations but with (nonlinear) coefficients that are not pure imaginary [1,2]. 
Spatially uniform steady solutions (i.e., standing waves) take the form $A=R \exp \mathrm{i} \phi_{A}, B=R \exp \mathrm{i} \phi_{B}$, where $R, \phi_{A}$ and $\phi_{B}$ are constants. It follows that

$$
\mu^{2}=1+\left(v-R^{2}\right)^{2}, \quad \mu \cos \left(\phi_{A}+\phi_{B}\right)=1
$$

(see Fig. 1c). The stability properties of this solution are determined from the dispersion relation for perturbations of the form

$$
A=R \mathrm{e}^{\mathrm{i} \phi_{\mathrm{A}}}(1+a), \quad B=R \mathrm{e}^{\mathrm{i} \phi_{B}}(1+b) .
$$

We find that the SW are stable with respect to spatially uniform perturbations for all $R$ when $v<0$ and for $R^{2}>v$ if $v>0$, and that they are unstable if $0<R^{2}<v$. This instability sets in along the straight line $R^{2}=v$ in Fig. 2 and corresponds to a saddle-node bifurcation (Fig. lc). Nonuniform perturbations with wave number $2 \pi n$ lead to the dispersion relation

$$
(s+1)^{2}+\left(R^{2}-v_{n}\right)^{2}+2 \beta R^{2}\left(R^{2}-v_{n}\right)=0,
$$

with $v_{n}$ defined in (44). Consequently the SW also lose stability along the curves

$$
1+\left(v_{n}-R^{2}\right)\left(v_{n}-(2 \beta+1) R^{2}\right)=0, \quad n=1,2, \ldots
$$

In view of the relation (44) these are hyperbolas in the $\left(v, R^{2}\right)$ plane. Fig. 2 summarizes the stability results in this plane, thereby avoiding complications arising from the presence of multiple SW in the $(v, \mu)$ plane (cf. Fig. Ic). Four distinct regimes are identified, depending on the sign of the dispersion coefficient $\alpha$ and the magnitude of the self-interaction cocfficient $\beta$ : (i) $2 \beta+1>0, \alpha>0$, (ii) $2 \beta+1<0, \alpha>0$, (iii) $2 \beta+1<0, \alpha<0$, (iv) $2 \beta+1>0, \alpha<0$. In cach case stable spatially uniform SW appear when $v=0$ ) and $R$ (equivalently $\mu$ ) increases.
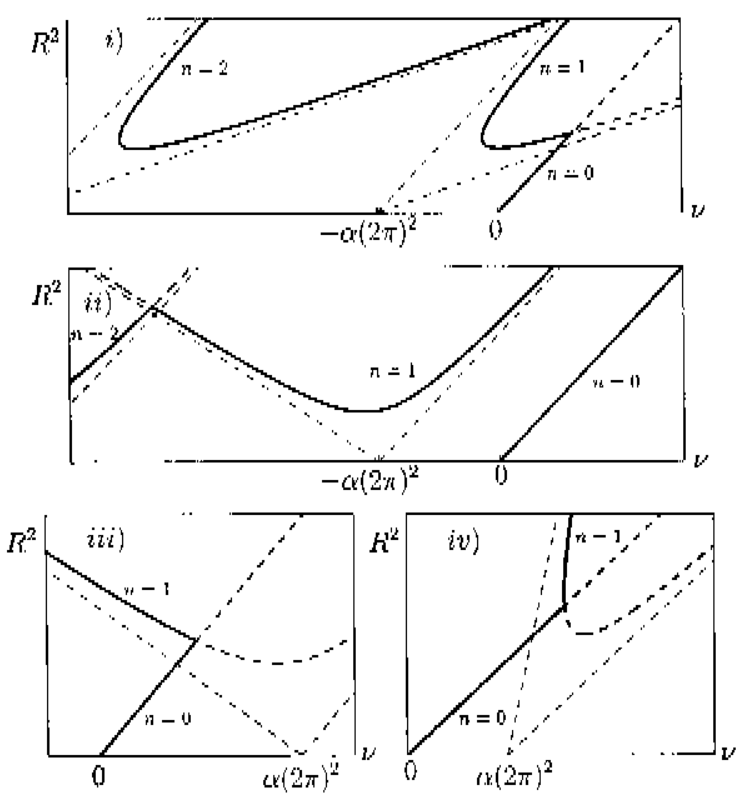

Fig. 2. Stability propertics of spatially uniform, steady solutions $A=B$ of Hqs. (38)-(40) for (i) $2 \beta+1>0$; $\alpha>0$, (ii) $2 \beta+1<0$, $\alpha>0$, (iii) $2 \beta+1<0, \alpha<0$ and (iv) $2 \beta+1>0, \alpha<0$. The shaded regions indicate instability; heavy lines indicate neutral stability curves, while the thin dashed lines show the asymptotes of the hyperbolas (44) and (49). 
This is because we are focusing on the $n=0$ instability tongue (see Fig. 1b). In the first three cases these spatially uniform SW lose stability with respect to spatially nonuniform perturbations as $R$ increases further. Note that these instabilities occur despite the fact that in the present scaling the corresponding instability tongues (9) are absent, i.e., without the nonlinear destabilization all nonuniform modes decay. However, as shown in Fig. 2, the small damping rate allows even small nonlinearities to excite such modes. Fig. 2 also shows that when $\alpha>0$ stability is lost to successively higher values of $n$ as $v$ decreases. Moreover, if $2 \beta+1>0$ the slopes of the asymptotes (thin dashed lines) of the hyperbolas (44) and (49) have the same sign, and hence for fixed $v$ the SW can lose and regain stability several times as $R^{2}$ (i.e., as the control parameter $\mu$ ) increases; however, if $2 \beta+1<0$ the SW never regain stability once they lose it. In contrast, if $\alpha<0$ the SW can only lose stability to spatially uniform perturbations or to nonuniform perturbations with $n=1$, and the stability boundary is the union of parts of the first hyperbola and the $n=0$ boundary. In this case, if $2 \beta+1<0$ the first hyperbola extends over all $\nu$ and the SW lose stability with increasing forcing $\mu$ regardless of $\nu$; on the other hand when $2 \beta+1>0$ all SW are stable at sufficiently large $\mu$.

The linear stability properties of the uniform solution $C=$ constant of Eqs. (41) and (42) are identical. However, as described in Section 4, the subsequent solutions of Eqs. (38)-(40), and of (41) and (42) need not be the same.

\subsection{Origin of drifts of type I}

In the results reported in Section 4 we distinguish two types of phase drifts, type I and type II. Both drifts have a simple and related origin as we now explain.

Consider a system that is equivariant under translations $x \rightarrow x+$ constant and reflection $x \rightarrow-x$. When periodic boundary conditions are imposed such a system has the symmetry $\mathrm{O}(2)$ of rotations and reflections of a circle. A primary steady state bifurcation from the trivial state produces a circle of nontrivial reflection-symmetric steady states distinguished by their spatial phase [17]. In the following we consider a secondary steady state bifurcation from such a circle of reflection-symmetric states, not necessarily of small amplitude. If this bifurcation breaks the reflection symmetry it is associated with a (steady) drift along the group orbit, i.e., a drift in spatial phase. Such a bifurcation is called a parity-breaking bifurcation. The resulting solution takes the form

$$
f_{a}(x, \tau)=f_{s}(x+\sigma)+c g_{a}(x+\sigma)+\cdots, \quad|c| \ll 1,
$$

where the subscripts $s$ and $a$ indicate functions that are symmetric and asymmetric with respect to reflection, and

$$
\dot{\sigma}=c+\cdots, \quad \dot{c}=\left(\mu-\mu_{d}-c^{2}\right) c+\cdots,
$$

cf. $[18,19]$. Here we have assumed that the secondary bifurcation takes place at $\mu_{d}$ and is supercritical. Thus when $c=0$ the solution is symmetric and does not drift; steadily drifting states are described by solutions with $c=$ $\pm \sqrt{\mu-\mu_{d}}$, the sign indicating the direction of the drift. Note that the drift direction is determined by the asy mmetry of the drifting state, and vice versa. The same behavior applies to parity-breaking bifurcation from a circle of standing waves, or more generally from a circle of strange attractors, with Floquet or Lyapunov exponents corresponding to nonreflection-symmetric cigenfunctions playing the role of the \%ero eigenvalue in the above discussion. In cach case the instability introduces a spatial drift into the dy namics and hence an additional frequency.

In Section 4 we cncounter drifts of this type in the solutions of both (38)-(40), and of (41) and (42), and identify their consequence for the dy namics of the reconstructed physical variable u defined by (1).

\subsection{Origin of drifts of type II}

To understand the origin of drifts of type II it is helpful to recexamine drifts of type I from a different perspective. At small amplitude (i.c., near onset of the primary instability) the symmetric function in (50) can be written in the 
form

$$
f_{s}(x)=A \mathrm{e}^{\mathrm{i} k x}+\text { c.c. },
$$

Consequently the translation and reflection equivariance responsible for the group orbit of reflection-symmetric solutions are equivalent to equivariance under

$$
\hat{\sigma}: A \rightarrow A \mathrm{c}^{\mathrm{i} \sigma}, \quad \hat{\kappa}: A \rightarrow \bar{A},
$$

and the parity-breaking bifurcation therefore generates a drift in the phase of the complex amplitude $A$. This way of looking at the symmetries of the problem makes contact with drifts of type II which arise when a bifurcation breaks the interchange symmetry $A \leftrightarrow B$ in Eqs. (38)-(40). In this case the relevant symmetries are given by (21) but these still constitute a representation of the group $\mathrm{O}(2)$. The symmetric (or $\hat{\kappa}$-invariant) solutions now correspond to states with $A=B$ (modulo translations, reflection and phase shifts), with the symmetry $\hat{\kappa}$ broken whenever $A \neq B$. In this case the solution of (38)-(40) near the symmetry breaking bifurcation is readily seen to be of the form

$$
A=\mathrm{e}^{\mathrm{i} \sigma}\left[A_{s}(\eta)+c a(\eta)+\cdots\right\rfloor, \quad B=\mathrm{c}^{-\mathrm{i} \sigma}\left[B_{s}(\xi)-c a(\xi)+\cdots\right],
$$

where $\left(A_{s}, B_{s}\right)$ is the basic symmetric solution, $(a(\eta),-a(\xi))$ is the marginally stable eigen-mode at the bifurcation point, and $\sigma$ and $c$ are real and given again by (51); note that $c=\mathcal{O}\left(|A|^{2}-|B|^{2}\right)$. Thus the phases of $A$ and $B$ will rotate in opposite directions.

The drifts just discussed came about as a result of a reflection-breaking steady state instability and were therefore time-independent. Symmetry-breaking Hopf bifurcations are also accompanied by drifts $[20\rceil$; however, since the resulting periodic orbit is symmetric with respect to $A=B$ the net drift vanishes. Consequently, net type II drift can be used as a diagnostic of the symmetry of the attractor. In fact drifts of both types may be present simultaneously. This is because the symmetry group of Eqs. (38)-(40) is the larger group $\mathrm{O}(2) \times \mathrm{O}(2) \times \mathrm{O}(2)$, where the first two symmetries come from translations and reflections in the comoving variables $\eta$ and $\xi$ and the third acts by (21). Bifurcations breaking any of the three possible reflection symmetries produce drifts by the mechanisms just described. To untangle the two types of drift it is convenient to focus on the phases of the $k=0, k=1$ and $k=-1$ Fourier components of each solution. Any drift in the phase of the $k=0$ component will be entirely of type II and hence due to asymmetry with respect to the invariant subspace $A=B$. Conversely, drifts in the phases of the $k= \pm 1$ components of $A$ and $B$ will be entirely due to type I drift provided $A=B$, cf. [21], but if $A \neq B$ the overall phase drift will be a superposition of both types of drift. In contrast, Eqs. (41) and (42) can only exhibit type I drifts that result from the $\mathrm{O}(2)$ symmetry associated with the invariance of (41) and (42) under spatial translations and reflection; this drift will be seen in the phases of the $k= \pm 1$ Fourier components of $C$.

\section{Results}

In this section we discuss detailed numerical solutions of both Eqs. (38)-(40) and of (41) and (42) and compare and contrast their behavior. The results are presented for zero detuning and for four sets of values of the remaining parameters, corresponding to the four regimes identified in Section 2 (see Fig. 2). In each case we vary the strength $\mu$ of the parametric forcing, corresponding to taking a particular vertical cut through the $(v, \mu)$ parameter plane shown in Fig. 2. We begin with Eqs. (41) and (42) which constitute the restriction of Eqs. (38)-(40) to the invariant subspace $A=B$ and summarize our results in the form of bifurcation diagrams. These are constructed by noting that Eqs. (41) and (42) imply

$$
\frac{\mathrm{d}}{\mathrm{d} \tau}\|C\|_{L_{2}}^{2}=-2\|C\|_{2}^{2}+\mu\left(\langle\bar{C}\rangle^{2}+\text { c.c. }\right)
$$


a)

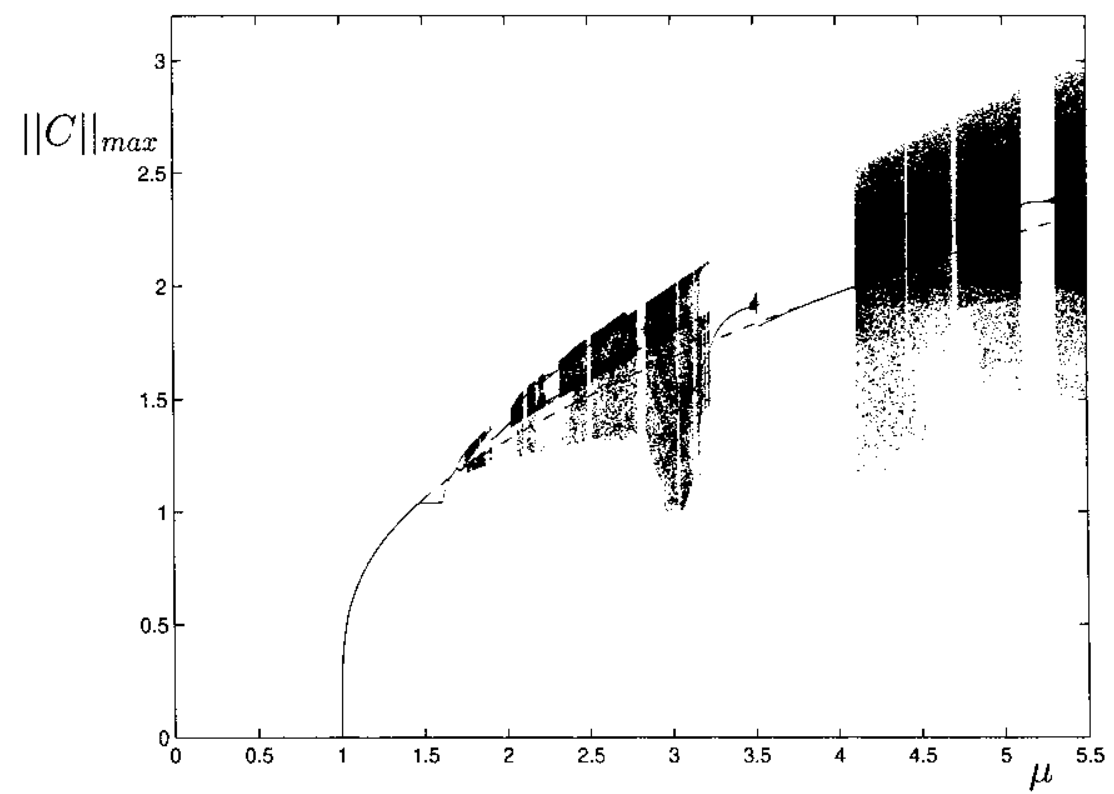

b)

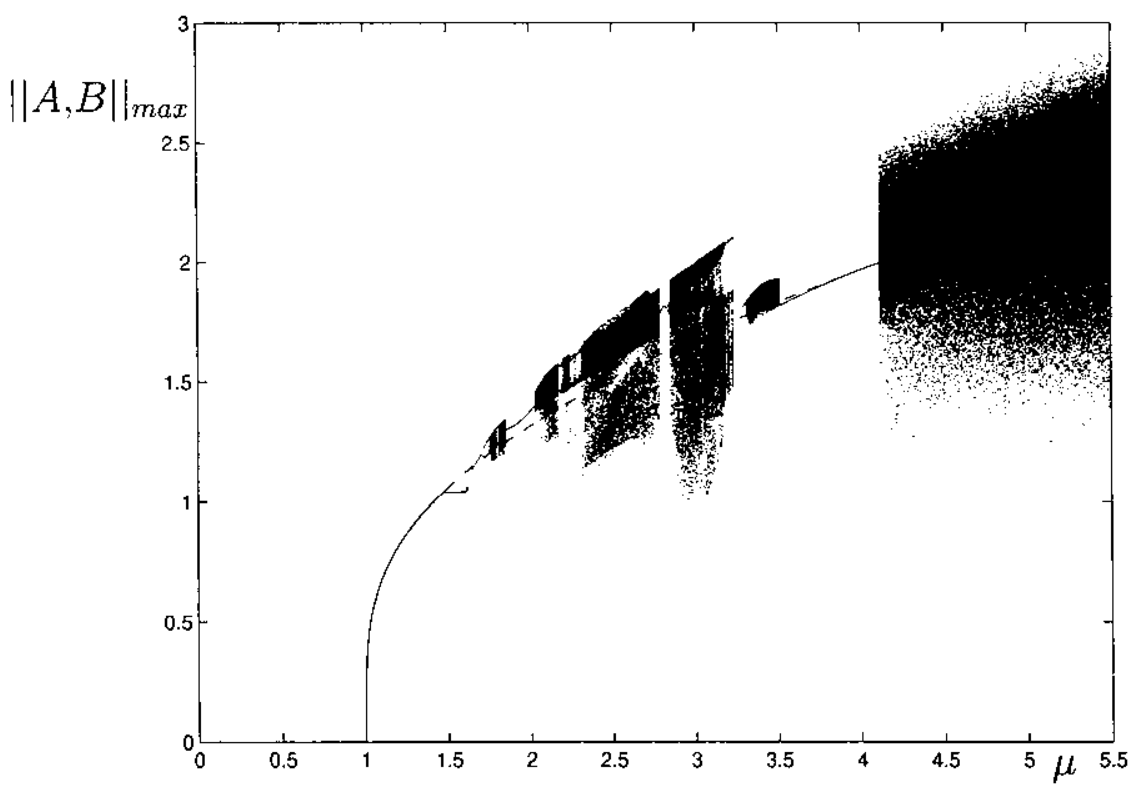

Fig. 3. Bifurcation diagrams for $\beta=1.5 . \alpha=0.1$ as a lunction of $\mu$ for (a) Eqs. (4I) and (42), and (b) Eqs. (38)-(40), both for $v=0$.

so that successive intersections of a trajectory with the hypersurface

$$
\|C\|_{L_{2}}^{2}=\frac{1}{2} \mu\left(\langle\bar{C}\rangle^{2}+\text { c.c. }\right),
$$

are always well defined. In fact this surface contains all steady states, while each periodic trajectory intersects it at least twice in each period, at the turning points in $\|C\|_{L_{2}}$. In the bifurcation diagrams we plot successive maxima 
a)
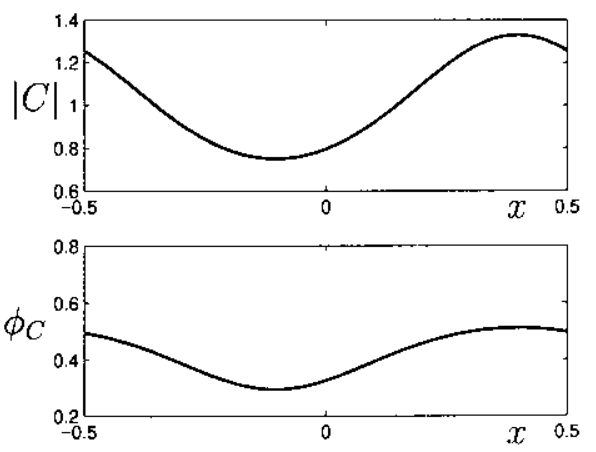

b)

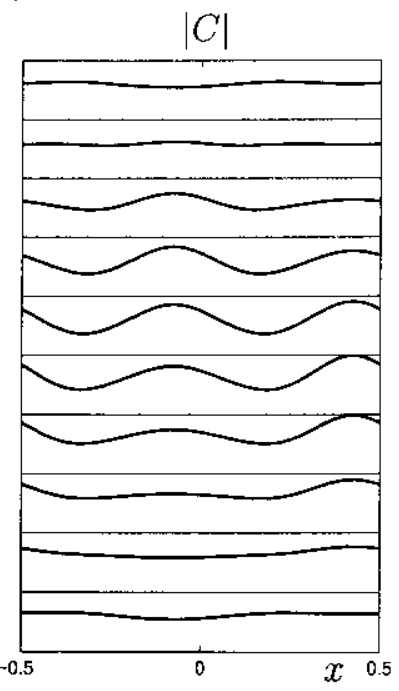

d) c)
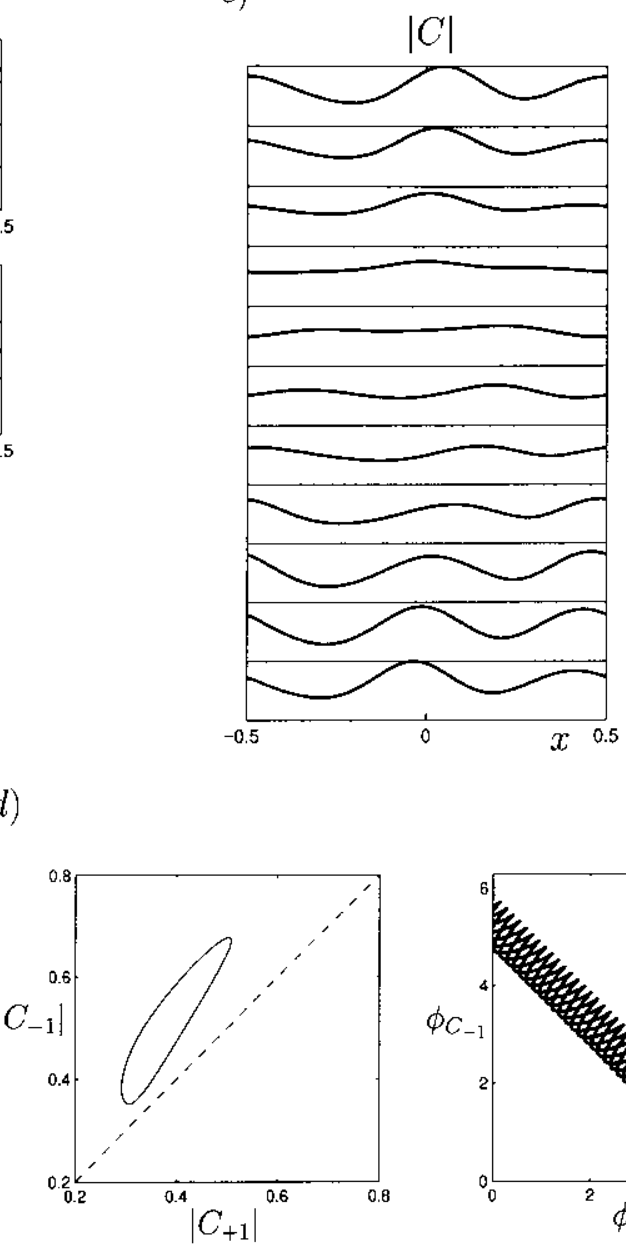

e)

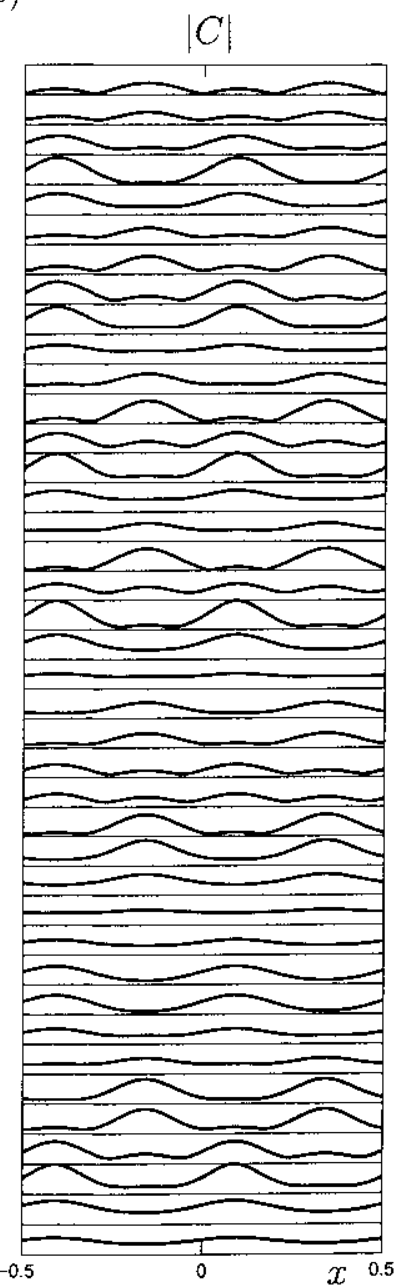

Fig. 4. Solutions $C(x, \tau)$ for the parameters of Fig. 3a when (a) $\mu=1.6$, (b) $\mu=3.3$, (c) $\mu=3.4$ and (e) $\mu=5.0$; (b), (c), and (e) show the spatial profiles of the solution at a sequence of equally spaced instants in $\tau$, with $\tau$ increasing upwards in increments ol 0.1 ; (d) shows that the solution (c) lacks spatial rellection symmetry and the associated drift in the phase difference $\phi_{C_{+1}}-\phi_{C_{-1}}$. This (rightward) spatial drift is slow and is not easily seen in the plot (c). 
a)

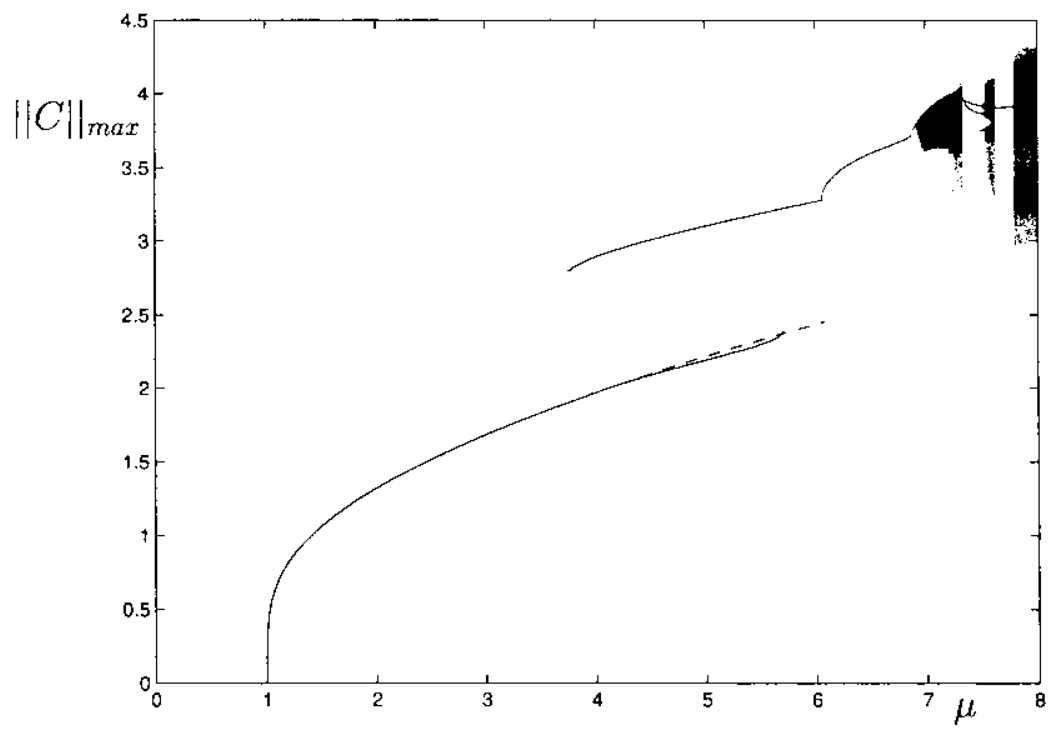

b)

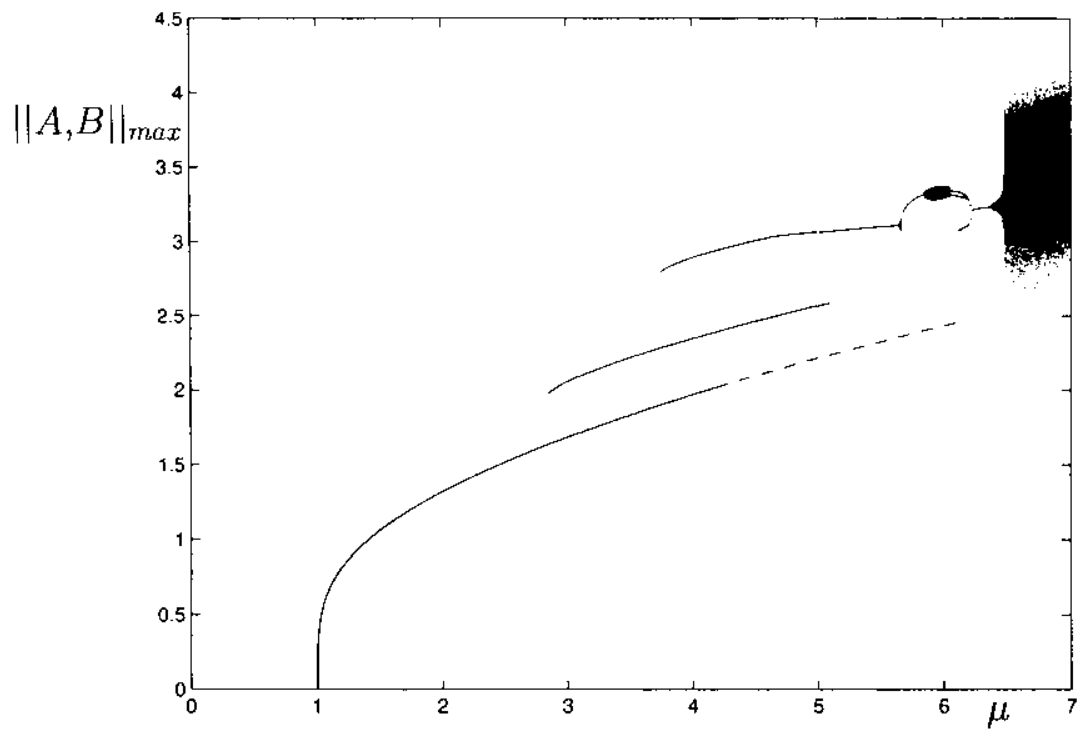

Fig. 5. Bifurcation diagrams for $\beta=-1.0 . \alpha=0.1$ as a function of $\mu$ for (a) Eqs. (4l) and (42), and (b) Eqs. (38)-(40), both for $v=0$.

of $\|C\|_{L_{2}}$ at each value of $\mu$ after transients have died away. In the general case we likewise plot the outward intersections with the hypersurface

$$
\|A\|_{L_{2}}^{2}+\|B\|_{L_{2}}^{2}=\mu(\langle\bar{A}\rangle\langle\bar{B}\rangle+\text { c.c. }) \text {, }
$$

corresponding to maxima in $\|A\|_{L_{2}}^{2}+\|B\|_{L_{2}}^{2}$. Although this procedure for generating bifurcation diagrams is convenient for most purposes it suffers from the disadvantage that it is insensitive to drifts of both type I and II. Thus additional diagnostics are necessary to identify such drifts. 


\subsection{Dynamics in the invariant subspace $A=B$}

In this section we describe the results of solving Eqs. (41) and (42) with $v=0$.

Case (i): We begin with the case $\beta=1.5, \alpha=0.1$. In this case the spatially uniform solution loses stability first at $\mu_{1}=1.47$ as the system enters the first instability tongue, but then regains it at $\mu_{2}=3.99$ when it exits the tongue before losing it again at $\mu_{3}=4.09$ when it enters the second instability tongue (see Fig. $2 \mathrm{i}$ ). In each tongue there are numerous regimes of chaotic dy namics interspersed with nonuniform but temporally periodic motion, as shown in Fig. 3a. Many of these transitions are the result of crises; others are due to cascades of period-doubling bifurcations. The first instability, at $\mu_{1}$, produces a time-independent but nonuniform state with reflection symmetry, i.e., both $|C(x)|$ and the phase of $C, \phi_{C}(x)$, are symmetric with respect to reflection $x \rightarrow x_{0}-x$ about the same point $x_{0}$ (see Fig. 4a). Owing to the periodic boundary conditions there is a whole circle of such solutions. By $\mu=1.7$ this solution has lost stability at a Hopf bifurcation to an oscillatory but still reflection-symmetric solution resembling the solution in Fig. 4a. Once again there is a whole circle of such solutions. After a number of intervals of chaos a stable periodic and reflection-symmetric solution is reestablished but with two maxima in the interval $0<x<1$ (see Fig. 4b). By $\mu=3.4$ the reflection symmetry is broken (Fig. $4 c$ ). Such asymmetric solutions are produced in steady state parity-breaking bifurcations as discussed in Section 3 and are accompanied by spatial drift, i.e., the solutions are quasiperiodic. For example, the solution shown in Fig. $4 \mathrm{c}$ is accompanied by rightward drift. However, both the asymmetry of the orbit and the spatial drift are more clearly seen in Fig. $4 \mathrm{~d}$, which shows the amplitudes and phases of the $k= \pm 1$ Fourier components of $c$; because of the spatial drift, the phases take the form $\phi_{C \pm 1}=\mp 2 \pi c \tau+g_{ \pm}(\tau)$, where $c$ is the net drift velocity and the $g_{ \pm}$are bounded. periodic functions of $\tau$. This solution becomes chaotic in a small interval followed by a (hysteretic) jump to a new time-independent reflection-symmetric state with two different maxima. After a second window with stable uniform SW the system enters the second instability tongue and becomes chaotic almost immediately; an example of a chaotic but reflection-symmetric solution in this tongue is shown in Fig. 4e for $\mu=5.0$.

Case (ii): When $\beta=-1.0, \alpha=0.1$ one samples a single instability tongue only (see Fig. 2ii). This tongue is entered at $\mu=\mu_{c} \approx 4.19$, where the uniform solution $|C|=$ constant loses stability (cf. Fig. 2ii). The resulting bifurcation diagram is shown in Fig. $5 \mathrm{a}$ and reveals the presence of substantial hysteresis between two solution branches both of which consist of time-independent and nonuniform solutions with reflection symmetry. Figs. 6a,b show typical solutions on the lower and upper branches, respectively. Given the similarity between these solutions it is likely that these two branches are in fact connected by an unstable branch of reflection-symmetric solutions (not shown in Fig. 5a); we have not, however, computed such unstable solutions. With increasing $\mu$ the upper branch undergoes a Hopf bifurcation producing reflection-symmetric oscillations, as shown in Fig. $6 \mathrm{c}$ for $\mu=6.5$. These oscillations are characterized by periodically occurring pairwise phase slips during which a half-wavelength is inserted at one location while at another location a half-wavelength is simultaneously removed. These pairwise phase slips preserve reflection symmetry and conserve the overall phase of the pattern in a process that is reminiscent of the double phase slips studied by Granzow and Riecke $[23,24]$. For $\mu$ slightly larger than these oscillations lose stability in a parity-breaking bifurcation and begin to drift. With increasing $\mu$ the solution acquires a third frequency (i.e., $C(x, \tau)$ becomes triply periodic) and then chaotic. The solution at $\mu=7.0$ (see Fig. $6 \mathrm{~d}$ ) is quasiperiodic, consisting of three frequencies one of which arises from spatial drift; both the asymmetry and the drift are clearly seen in the plots of the $k= \pm 1$ Fourier components in Fig. Ge. For larger $\mu$ the bifurcation diagram shows several intervals of two-frequency $C(x, \tau)$ (one frequency being the associated drift frequency) interspersed with regions of chaos. Finally, Figs. 6f,g show the symmetric chaotic attractor found when $\mu=8.0$; the corresponding solutions exhibit instantaneous drift, but because of the symmetry of the attractor there is no net drift over long times.

Case (iii): When $\beta=-1.0, \alpha=-0.1$ the situation is quite different. For these parameter values the instability of the uniform solution at $\mu_{c}=4.19$ results in a finite amplitude (but apparently nonhysteretic) jump into a 
a)
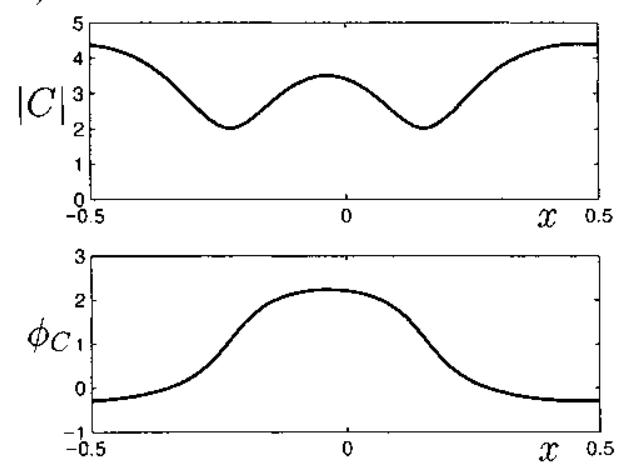

c)

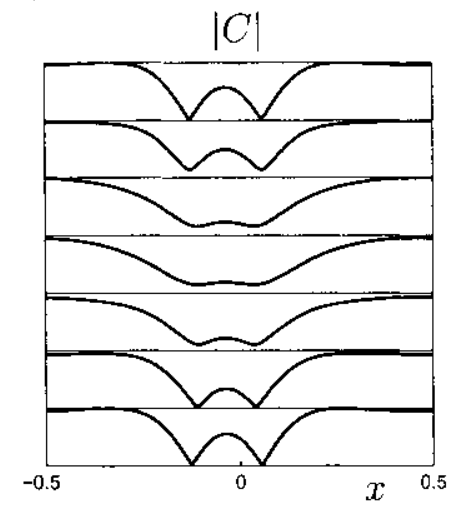

b)
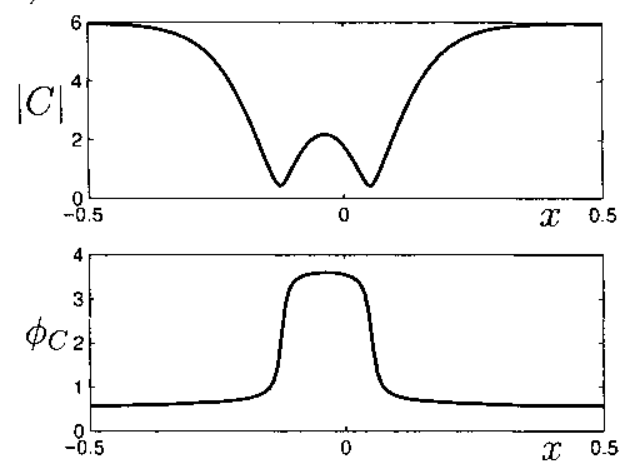

d)

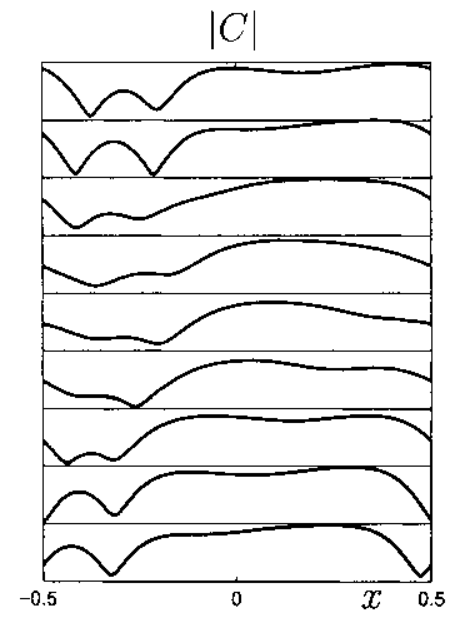

Fig. 6. As for Fig. 4 but for the parameters of Fig. 5 a when (a) $\mu=5.7$, (b) $\mu=6.0$. (c) $\mu=6.5$, (d) $\mu=7.0$ and (f) $\mu=8.0$. In (c) phase slips occur in pairs because of the reflection symmetry of the solution; this is no longer true in (d) or (f): (e) and (g) show projections of the attractors on the amplitudes of the $k= \pm 1$ Fourier components corresponding to (d) and ( $)$, and the corresponding time series for the phase difference $\phi_{C_{+1}}-\phi_{C_{-1}}$, relative to an arbitrary origin. In the former the attractor is asymmetric and a net drift is present; in the latter the attractor is symmetric and there is no net drift.

chaotic state (see Fig. 7a), presumably as a result of a crisis bifurcation. These solutions represent chaotic standing waves within Eqs. (41) and (42), i.e., these waves retain reflection symmetry (see Fig. 8a). The dynamics in this regime are very similar to those described recently by Bayliss and Matkowsky [22] in the context of solid fuel combustion. Specifically, at $\mu=4.5$ a large amplitude solitary wave splits symmetrically into two smaller waves that propagate in opposite directions and these collide again after traveling half the length of the domain, reconstituting the original wave. By $\mu=5.5$ the solution has lost its reflection symmetry and the chaotic oscillations are now accompanied by a chaotic drift (see Fig. 8b). Solutions of this type are not reported by Bayliss and Matkowsky.

It is of interest to compare the behavior in regions (ii) and (iii). These differ only in the sign of the dispersion term. In both cases one finds solutions resembling solitary waves, depression waves (dark solitary waves) in case (ii) (e.g., Fig. 6f) and elevation waves (bright solitary waves) in case (iii) (e.g., Fig. 8b). The dominant mechanism governing the evolution in the former case appears to be annihilation of neighboring solitary waves, 
e)

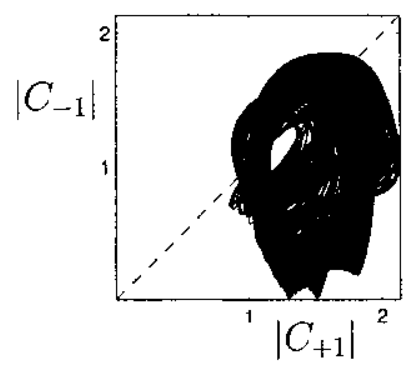

$$
\phi_{C_{+1}}-\phi_{C_{-1}}
$$

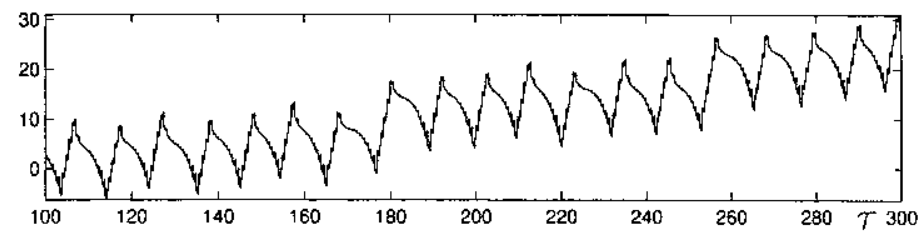

g)
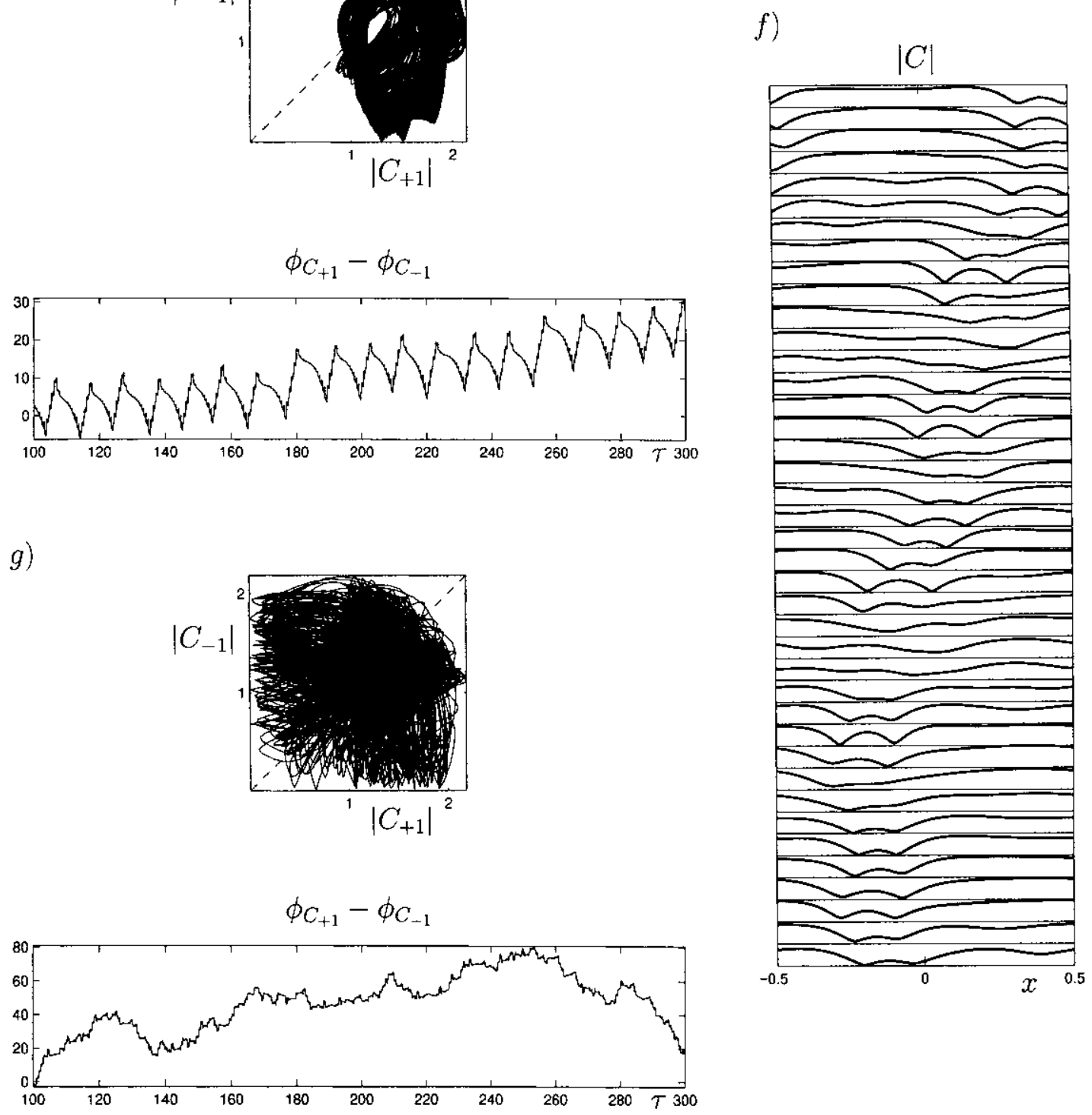

Fig. 6. (Contimued).

with their spontaneous creation elsewhere, while in the latter case the evolution is driven by splitting events, followed by reconstitution. In either case the observed solitary waves resemble those present in the damped nonlinear Schrödinger (NLS) equation with local parametric forcing [25] with the nonlocal nonlinear term playing the role of detuning.

Case (iv): $2 \beta+1>0, \alpha<0$. We have not found any stable spatially nonuniform or time-dependent states in this case. 
a)

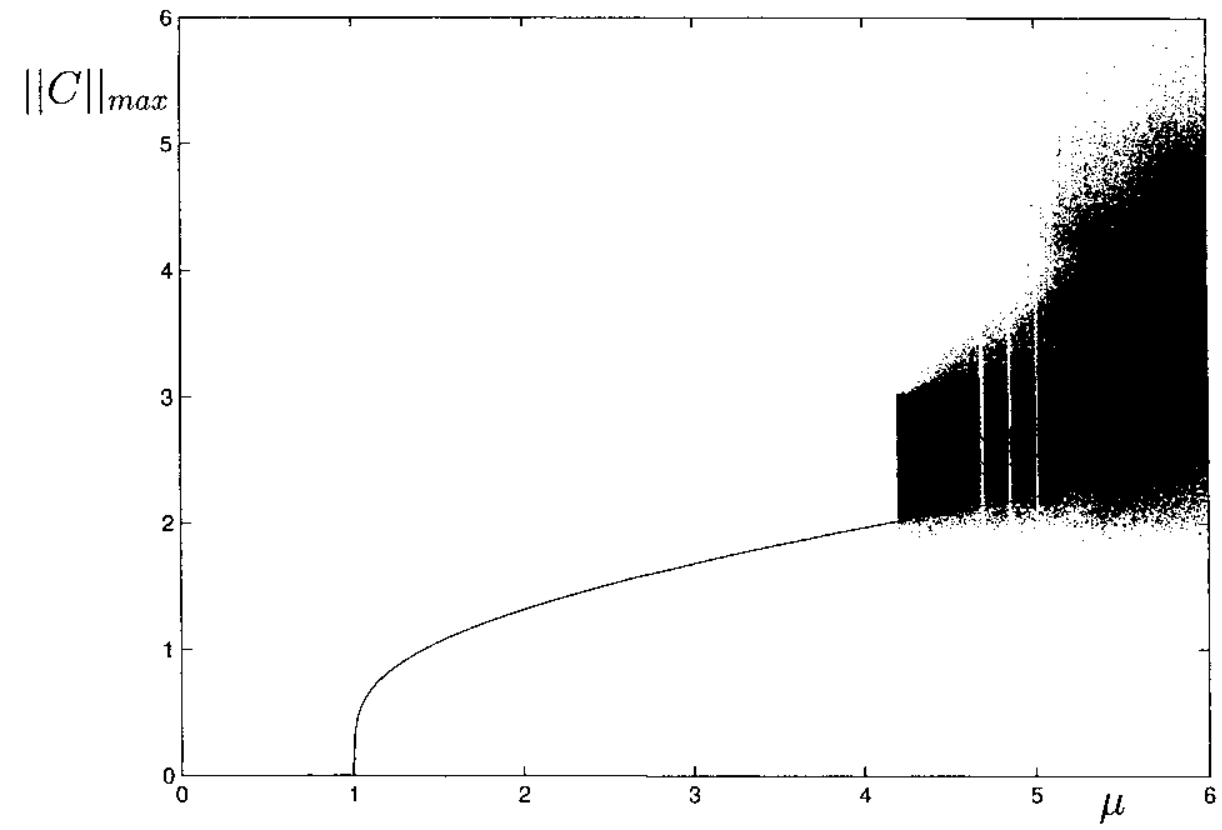

b)

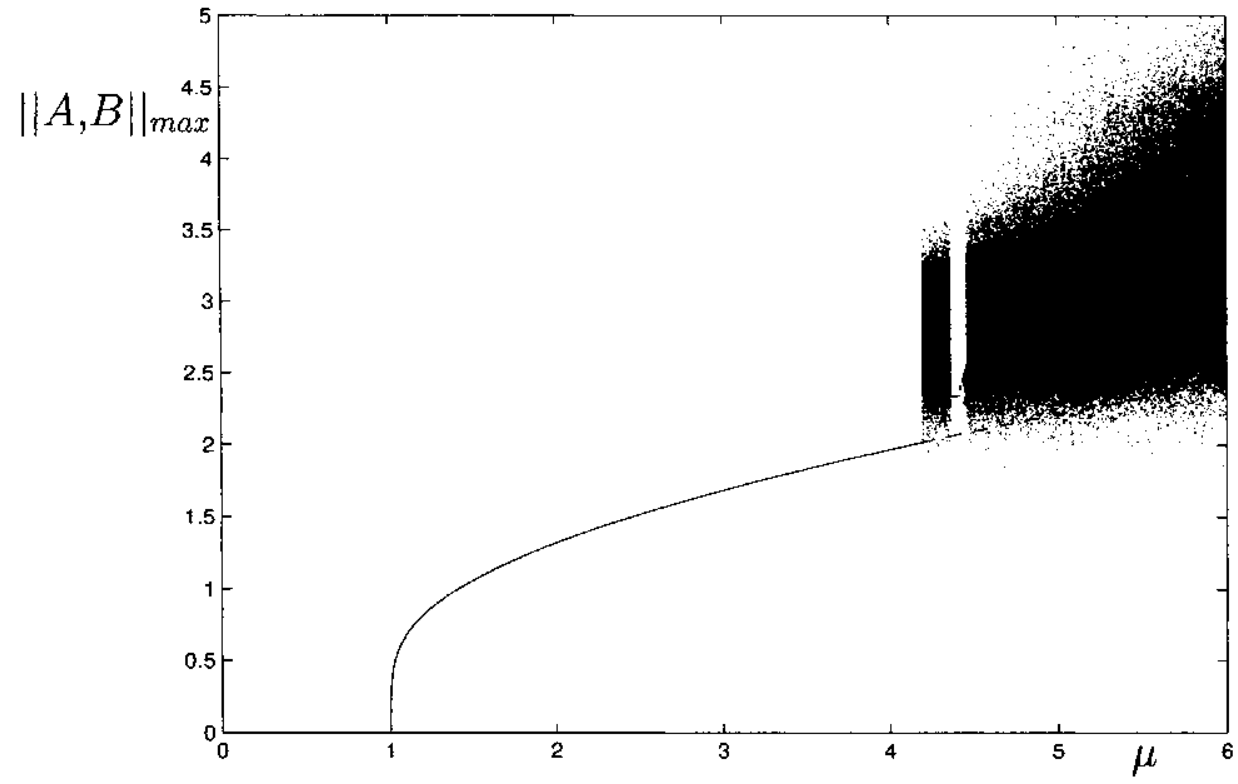

Fig. 7. Bifureation diagrams for $\beta=-1.0, \alpha=-0.1$ as a function of $\mu$ for (a) Eqs. (41) and (42), and (b) Eqs. (38)-(40), both for $v=0$. 
a)

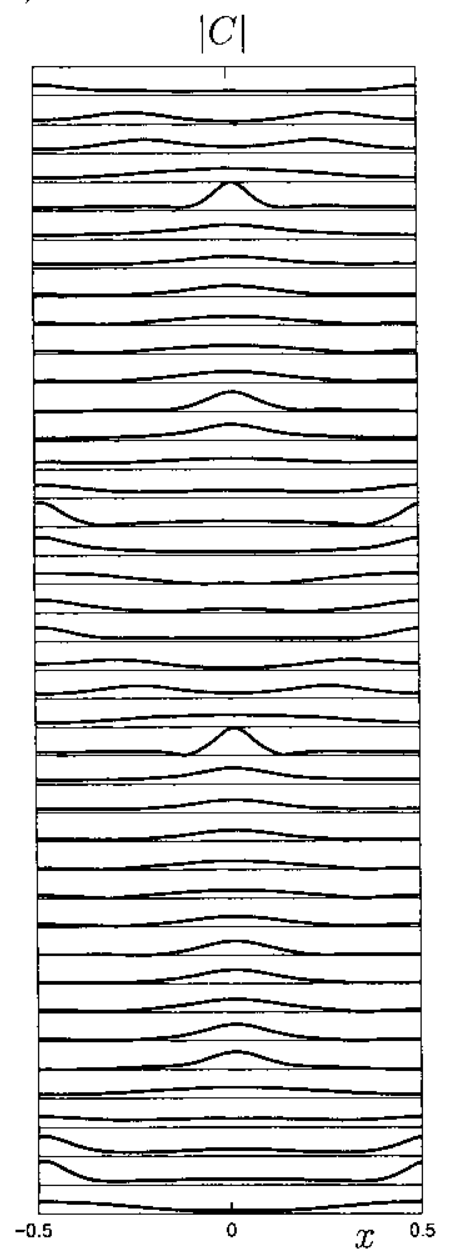

b)

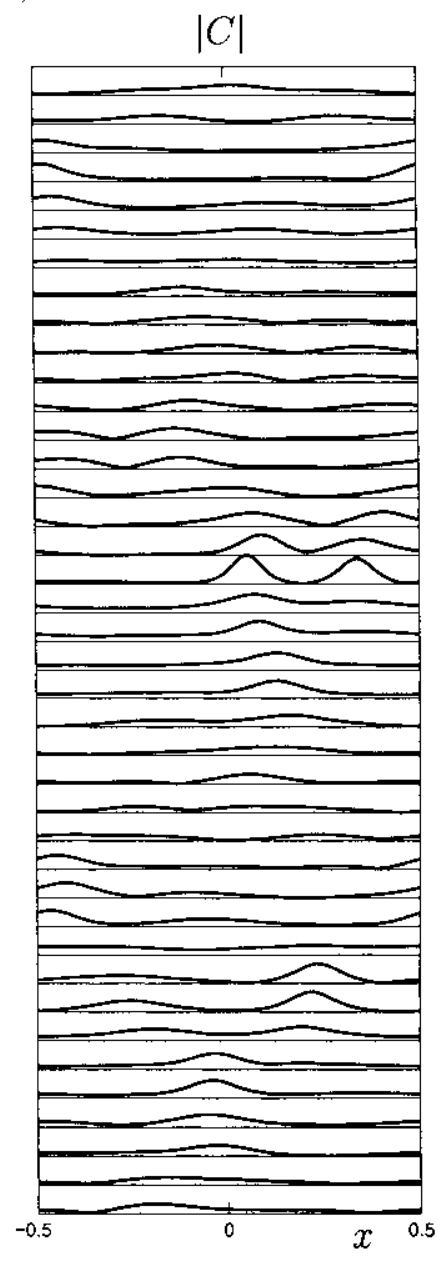

Fig. 8. As for Fig. 4 but for the parameters of Fig. 7a when (a) $\mu=4.5$, and (b) $\mu=5.5$.

\subsection{Dynamics in the general case}

We now discuss the results of integrating the more general equations (38)-(40) in the same four regimes. Both amplitudes are shown as a function of a variable $x$, where $x$ stands for $\eta \equiv x+t$ if the amplitude in question is $A$ or $\xi \equiv x-t$ if the amplitude is $B$. Consequently, the entire time-dependence of $A, B$ in the time series shown below arises from their dependence on the slow time $\tau$. Note that despite the possibility that $A \neq B$ the $\mathrm{L}^{2}$ norms of these amplitudes on the attractor are always identical.

(i) $\beta=1.5, \alpha=0.1$. The bifurcation diagram obtained by increasing $\mu$ is shown in Fig. $3 \mathrm{~b}$. The diagram bears a superficial resemblence to that shown in Fig. 3a but a more detailed look reveals a number of differences. These are due to the presence of new types of solutions with $A \neq B$ to which solutions with $A=B$ can become unstable; because of (20) and (21), the condition $A=B$ is defined hereafter modulo spatial translations, reflection and phase shifts. The results show that the initial instability at $\mu_{1}$ continues to be to a state in the invariant subspace $A=B$. This solution is initially time-independent and then undergoes a Hopf bifurcation, as in Eqs. (41) and (42). A sequence of period-doubling bifurcations leads to chaos but the ensuing chaotic interval already shows some 
a)

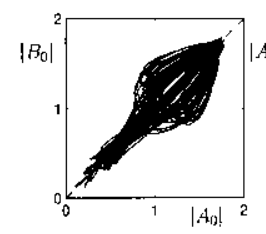

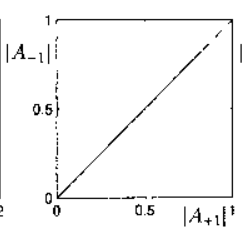
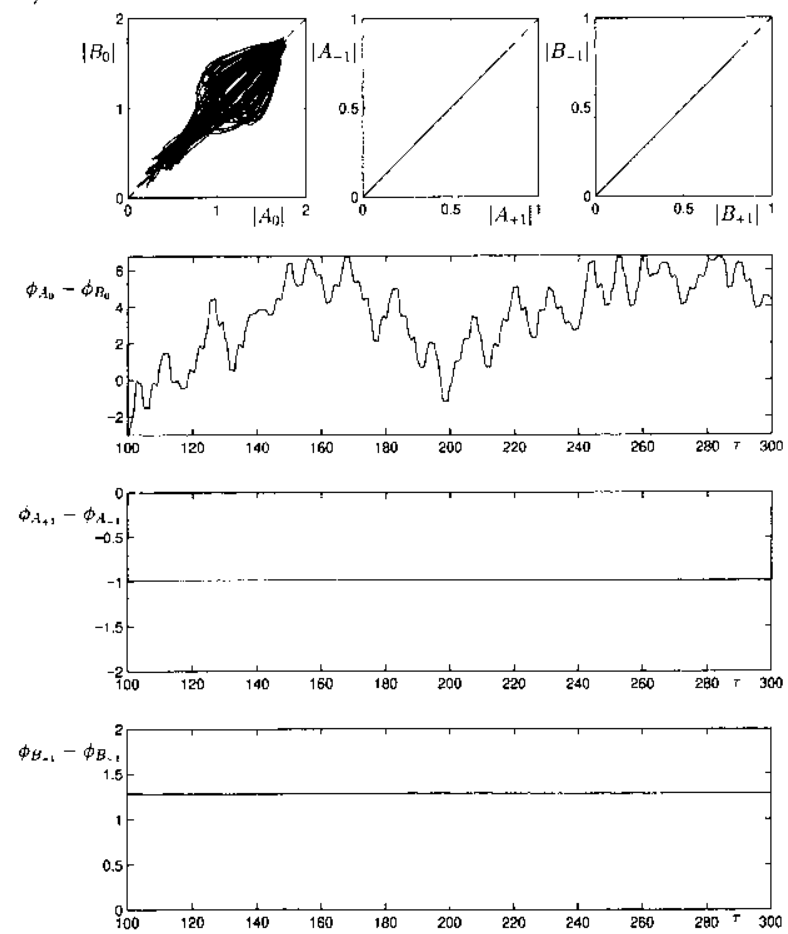

b)
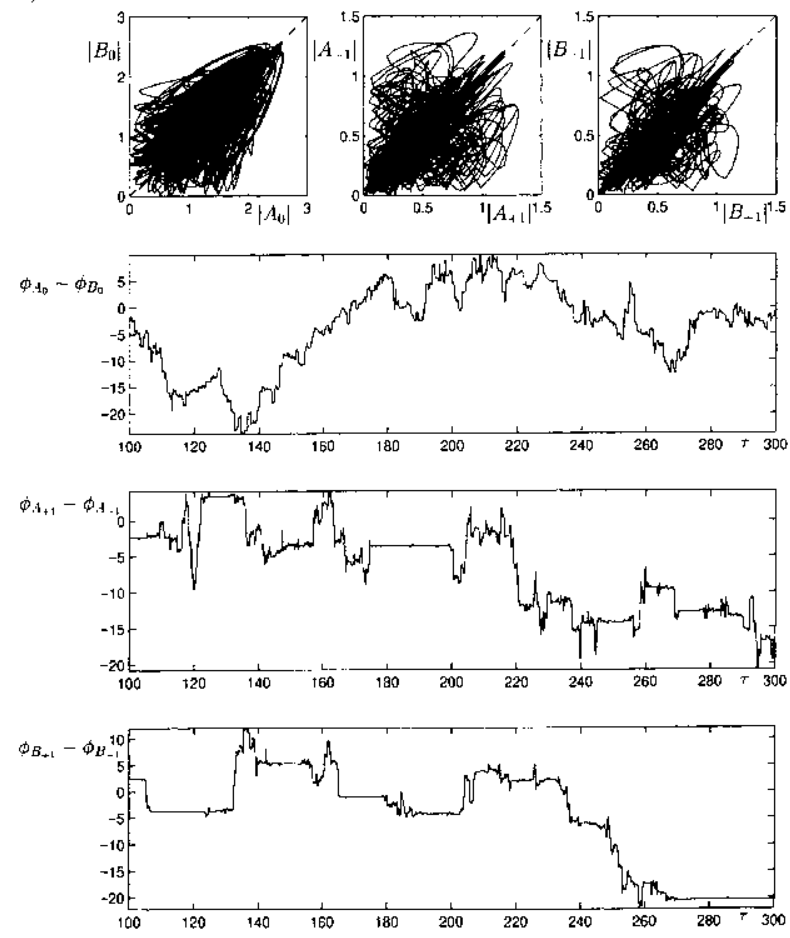

Fig. 9. Solutions $A(x, \tau) . B(x, \tau)$ for the parameters of Fig. 3b when (a) $\mu=2.6$, and (b) $\mu=5.0$, projected onto the $k=0$, \pm 1 Fouricr components. The attractor in (a) is symmetric under interchange of $A . B$ and the solutions are reflection-symmetric. In contrast. in (b) the reflection symmetry is broken but remains on average, with $A$ and $B$ both spending significant periods in nearly reflection-symmetric states: (c) shows an example of the time evolution of the amplitudes for case (b) in which both $A(x, \tau)$ and $B(x, \tau)$ are simultancously nearly symmetric. with $\tau$ increasing upwards in increments of 0.1 ; (d) shows the spatial structure at a particular instant in time, with solid (dashed) lines indicating $A(B)$.

differences from that shown in Fig. 3a. The oscillatory solution that emerges from this chaotic window again lies in the invariant subspace $A=B$ and hence is identical to that in Fig. 3a. The chaotic dynamics that follow for larger $\mu$ remain initially in this subspace but with increasing $\mu$ lose stability to solutions breaking the interchange symmetry. We show an example in Fig. 9a for $\mu=2.6$. The figure shows the dynamics of the (complex) Fourier amplitudes $A_{0}(\tau), B_{0}(\tau)$ of the $k=0$ components of $A$ and $B$ which we use as a diagnostic of the instantaneous solution symmetry with respect to the invariant plane $A=B$ and hence of the presence of type II drift. The lack of instantaneous symmetry translates into an instantaneous drift in the phase difference $\phi_{A_{0}}-\phi_{B_{0}}$; however, because of the interchange symmetry of the attractor (Fig. 9a) there is no net or overall drift in $\phi_{A_{0}}-\phi_{B_{0}}$, much as there is no net drift associated with a reflection symmetry-breaking Hopf bifurcation from a circle of steady states eventhough the resulting pattern drifts back and forth [20]. Fig. 9a also shows repeated periods of time during which the instantaneous drift is absent and $\phi_{A_{0}}+\phi_{B_{0}}$ increases instead. These periods correspond to motion near the $A=B$ invariant subspace; the time series for $\phi_{A_{0}}-\phi_{B_{0}}$ shows clearly the resulting periods of approximately constant phase difference. The figure suggests that the solution trajectory spirals within this subspace before being ejected in a direction normal to it. These ejections out of the invariant subspace appear to occur intermittently, much as might be expected of dynamics associated with a blow-out bifurcation and the so-called in-out intermittency [27,28]; similar behavior occurs in other partial differential equations with reflection-invariant subspaces such as those studied by Proctor and Lega [29] or Covas et al. [30]. Also included in Fig. 9a is a plot of $\left|A_{+1}\right|$ vs. $\left|A_{-1}\right|$ and of the associated 
c)
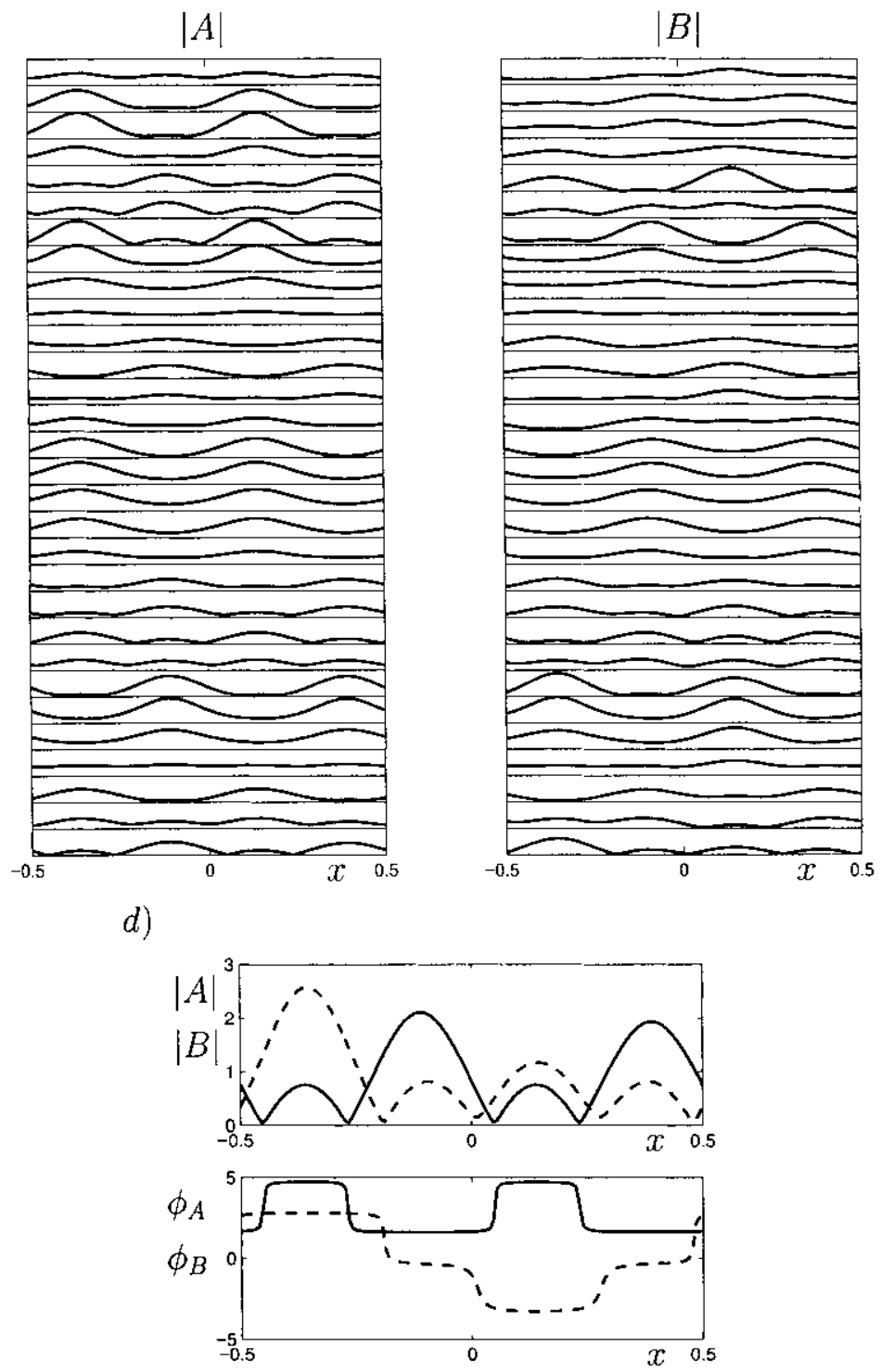

Fig. 9. (Contimied).

phases. We use the former as a diagnostic of the spatial symmetry of the solution; in the present case the solution has spatial reflection symmetry since $\left|A_{+1}\right|=\left|A_{-1}\right|$ for all $\tau$. The absence of type I drift is confirmed by the absence of a drift in the phase difference $\phi_{A_{11}}-\phi_{A}$; the drift in their sum reflects temporal evolution. At $\mu=3.27$ the solution is again periodic and lies in the invariant subspace; it is still reflection-symmetric. After an interval of chaos involving states with $A \neq B$ a periodic solution with $A=B$ acquires stability and subsequently disappears in a Hopf bifurcation. At $\mu=3.65$ the stable solution lies in the invariant subspace and is time-independent but still nonuniform (cf. Fig. 3a).

The dynamics in the second instability tongue are almost entirely chaotic; even the periodic windows visible in Fig. 3a are now absent. We show an example in Fig. 9b. For this solution $A \neq B$ and spatial reflection symmetry 
b)

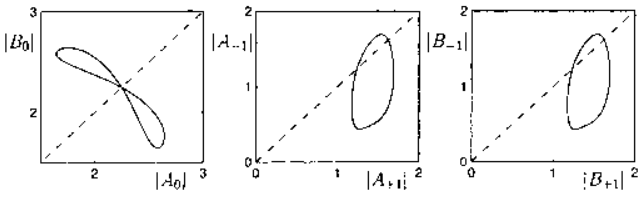

a)
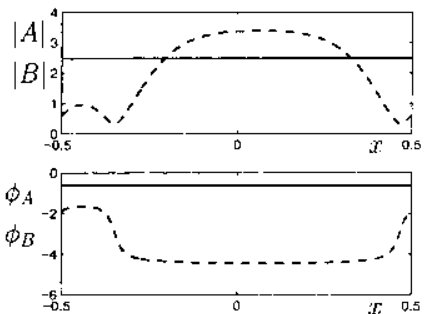

c)

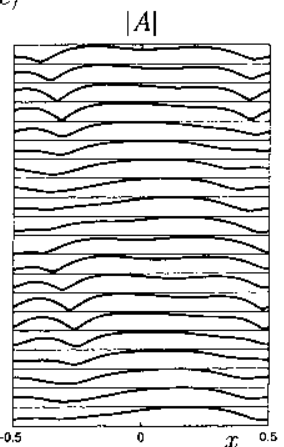

$|B|$

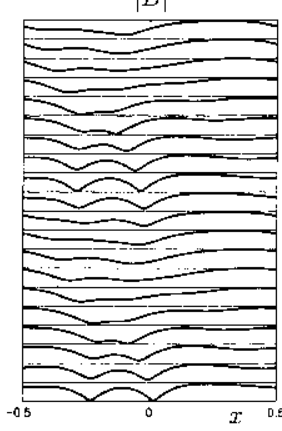

$\phi_{A_{u}}-\phi_{D_{\mathrm{u}}}^{2}$
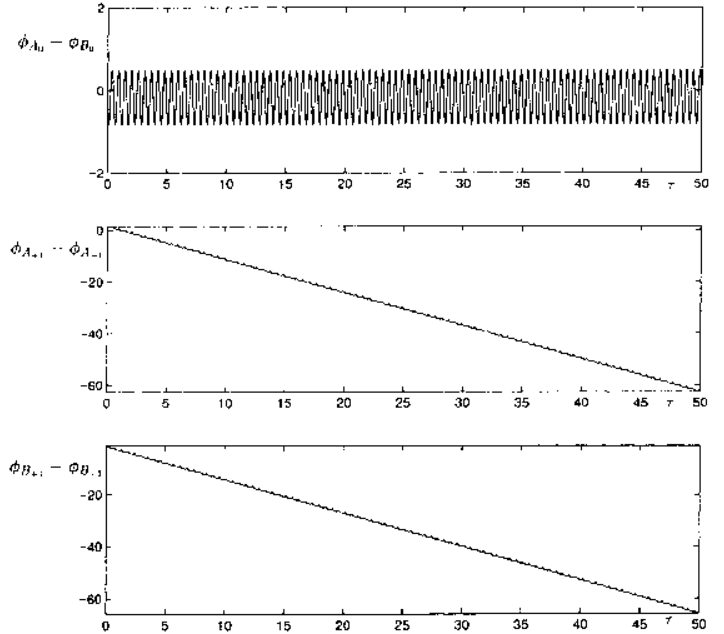

d)
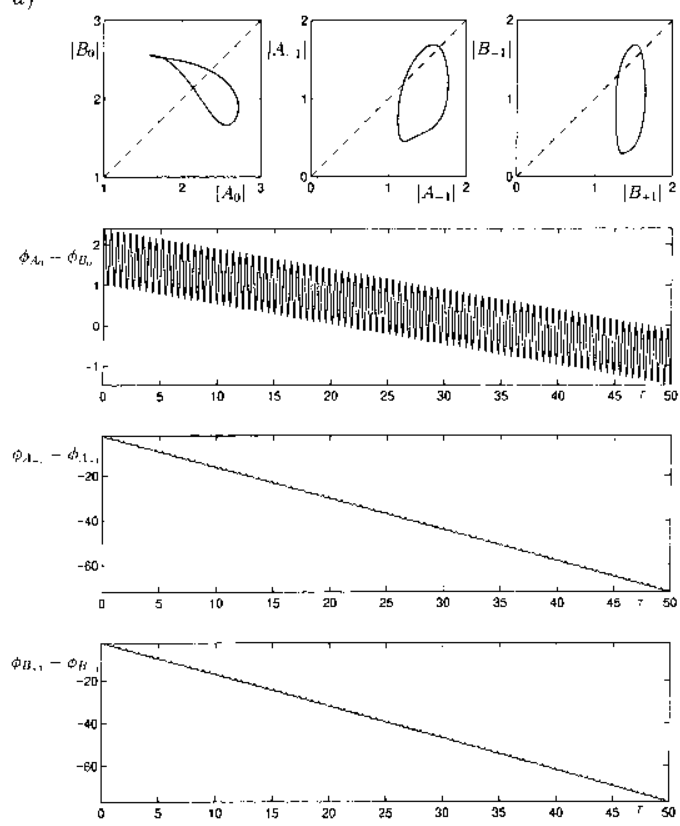

Fig. 10. As for Fig. 9 but for the parameters of Fig. 5b. (a) Amplitude profiles when $\mu=4.6$. (b) Altractor projections on the $k=0 . \pm 1$ Fourier modes for $\mu=5.6$. showing net type I drift but the absence of net type 11 drift: the corresponding evolution of the spatial profiles of the amplitudes is plotted in (c): (d). (e), and (f) show the projections on the $k=0 . \pm 1$ modes when $\mu=5.67 . \mu=6.0$ and $\mu=6.7$. In (d) and (c) the interchange symmetry is broken and a net type II drift has also developed. This drift has two frequencies in (d) and three in (c). In (f) the attractor is symmetric under interchange and the solution shows (sometimes very) long but intermittent trapping in reflection-invariant subspaces; no net drift of either type is present. 
is also broken, as shown by the $\left|A_{0}\right|$ vs $\left|B_{0}\right|$ and $\left|A_{+1}\right|$ vs $\left|A_{-1}\right|$ plots, respectively. Consequently, instantaneous drifts of both types are present and these can be discerned from the corresponding phase plots; note that both types of drift affect the phases of $A_{ \pm 1}$ and $B_{ \pm 1}$. However, the amplitude plots also show that the solution has both an interchange symmetry and a reflection symmetry on average, cf. [26]. Consequently, there can be no net drifts of either type. In fact both $A(x, \tau)$ and $B(x, \tau)$ spend much time near the state with spatial reflection symmetry. During these periods the instantaneous type I drifts disappear also and the corresponding phases drift along the $45^{\circ}$ line. This is confirmed by the time series for $\phi_{A_{11}}-\phi_{A}$, and $\phi_{B .1}-\phi_{B}$, which show prolonged intervals during which either $A$ or $B$ is almost exactly reflection-symmetric (zero instantaneous drift). The time series also show that typically this does not happen simultaneously for both amplitudes, i.e., that the interchange symmetry is almost always broken. This conclusion is confirmed by the time series for $\phi_{A_{0}}-\phi_{B_{0}}$. Nonetheless, it is possible to find time intervals during which both $A(x, \tau)$ and $B(x, \tau)$ are simultaneously almost reflection-symmetric (Fig. $9 c)$. Such sporadic excursions from the reflection-invariant subspaces are again likely to be the consequence of a blow-out bifurcation [27,28]. Fig. $9 \mathrm{~d}$ shows typical profiles of the associated phases $\phi_{A, B}(x)$ in this regime. These have a "square-wave" appearance due to the addition or subtraction of half a wavelength when either amplitude passes through zero. These phase slips typically occur in pairs but do not occur simultaneously in both amplitudes. Similar but time-independent solutions are shown in Fig. 6 b.

(ii) The bifurcation diagram for the case $\beta=-1.0, \alpha=0.1$ is shown in Fig. $5 \mathrm{~b}$ and reveals substantial differences from the corresponding diagram in Fig. 5a. The dominant difference is the presence of a "middle" branch of reflection-symmetric solutions that break interchange symmetry. These solutions have the special form $A=\hat{A} \exp (2 \pi \mathrm{i} c \tau), B=\hat{B}(x) \exp (-2 \pi \mathrm{i} c \tau)$, where $\hat{A}, \hat{B}$ are different and time-independent (Fig. 10a). As a

e)
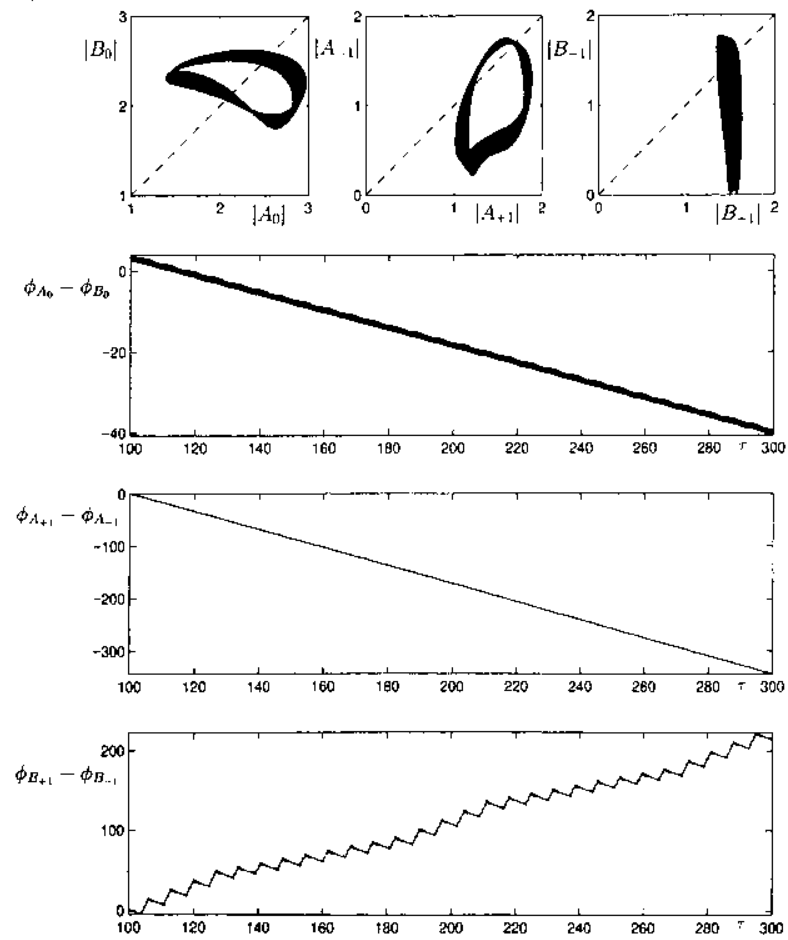

f)
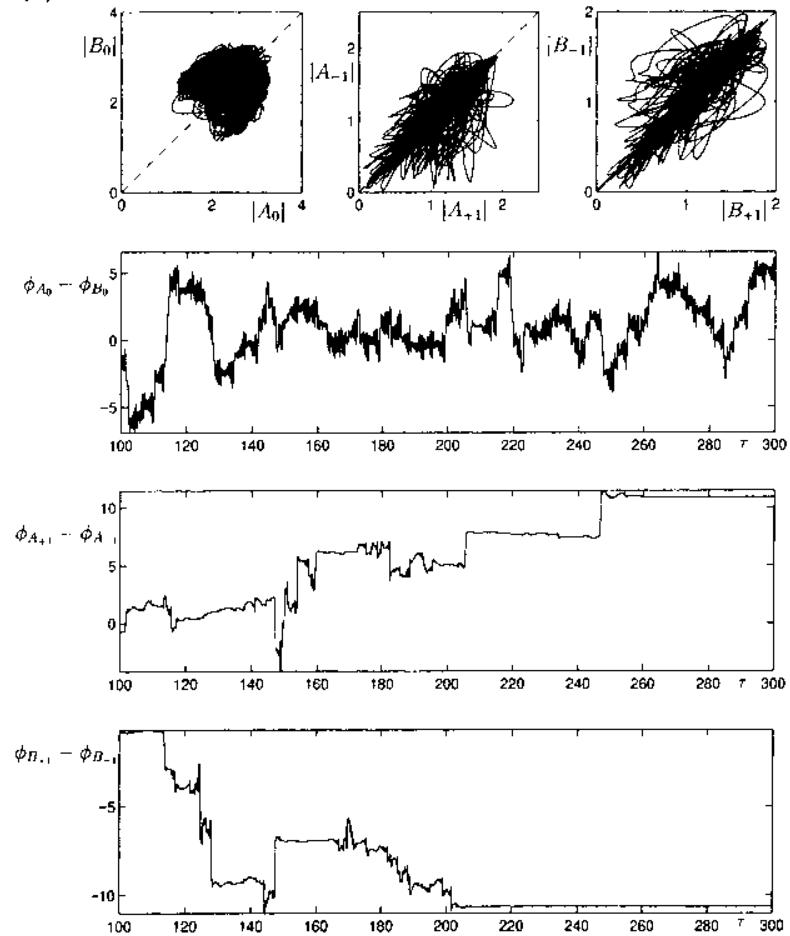

Fig. 10. (Continued) 
result the associated type II drift is constant in time. When the primary uniform solution loses stability the system now makes a transition to the middle branch, followed by a jump to the upper branch where $A=B$ (see Fig. 6b) as $\mu$ is increased further. When $\mu$ is decreased sufficiently far on either the middle or the upper branch the original spatially uniform solution is recovered. Thus the presence of the middle branch alters dramatically the nature of the hysteresis loop shown in Fig. 5a.

A further significant difference concerns the location of the Hopf bifurcation on the upper branch. This bifurcation now occurs at $\mu \approx 4.7$, i.e., substantially earlier than in Fig. 4 a, and involves a Hopf bifurcation out of the invariant subspace $A=B$. Such a bifurcation generates oscillations in $A, B$ that are symmetric under interchange of $A$ and $B$. The associated phases therefore oscillate about zero, but there is no overall type II drift [20]. Moreover, the bifurcated solution retains its spatial reflection symmetry, and hence no type I drift is present. But as $\mu$ is increased, the spatial reflection symmetry of the periodic solution is lost, and both $A$ and $B$ begin to drift, as shown in Figs. $10 \mathrm{~b}, \mathrm{c}$ for $\mu=5.6$. For this solution $A$ and $B$ are half a period out of phase; consequently, the phases of $\Lambda_{ \pm 1}$ undergo a two-frequency drift, a type I drift with a net mean due to the spatial asymmetry of the solution and a type II drift with zero mean due to the oscillation about $A=B$ (see Fig. 10b). Note that, because of the symmetries (20), at $\mu=5.6$ the system exhibits four different attractors (all 2-tori), two of which preserve the symmetry (23) and thus yield reflection-symmetric solutions of Eqs. (5)-(7), while the other two break this symmetry. With increasing $\mu$ the oscillation about $A=B$ becomes asymmetric as shown in Fig. $10 \mathrm{~d}$ for $\mu=5.67$ and the phases acquire net type II drift, in addition to the net type I drift already present (compare the $\left(\phi_{\Lambda 0}, \phi_{B 0}\right)$ plot with the corresponding plot in Fig. 10b); because of the reflection symmetries (20) and (21), this solution in fact generates eight distinct attracting 3-tori as solutions of Eqs. (38)-(40). None of these attractors preserve the symmetry (23) and thus all of them correspond to non-symmetric solutions of Eqs. (5)-(7). By $\mu=6.0$ this solution has likely undergone a Hopf bifurcation to what appears to be a quasiperiodic state (Fig. 10e). This quasiperiodic state lacks both the spatial reflection symmetry and the interchange symmetry and hence exhibits net drifts of both type I and type II. The resulting state has four independent frequencies; the reflection symmetries therefore generate eight distinct 4-tori. As $\mu$ is increased further, the solution undergoes several additional transitions. A chaotic solution with chaotic type I and type II drifts is shown in Fig. 10 for $\mu=6.7$. The corresponding attractor is likely both interchange-and reflection-symmetric and hence no net drifts of either type are expected. A plot of $\phi_{B_{0}}-\phi_{\Lambda_{0}}$ as a function of $\tau$ (Fig. 10f) confirms this expectation. Note, however, that despite the chaotic motion about the $A=B$ subspace both $\Lambda(x, \tau)$ and $B(x, \tau)$ spend sometimes quite long periods of time very close to reflection-invariant subspaces before escaping again. As already mentioned, behavior of this type may be associated with the onset of in-out intermittency $[27,28]$. This behavior is not revealed unambiguously by time series shown in Fig. 10 f because the segment shown is too short relative to ty pical "Irapping" (imes which may last several hundred time units.

(iii) The bifurcation diagram for $\beta=-1.0, \alpha=-0.1$ is shown in Fig. $7 \mathrm{~b}$. According to the lincar stability results the uniform state remains stable until $\mu=4.19$, where there is an apparently nonlysteretic transition to chaos. As before, we associate this transition with a crisis. However, there are again differences between the bifurcation diagrams of Figs. 7a,b. Fig. $7 \mathrm{~b}$ reveals the presence near $\mu=4.4$ of a window of time-independent but nonuniform solutions with $A \neq B$ that replaces the chaolic solutions found in Fig. 7a. These solutions take the special form $A=\hat{A}(x) \exp (2 \pi \mathrm{i} c \tau), B=\hat{B}(x) \exp (-2 \pi \mathrm{i} c \tau)$, where $\hat{A}, \hat{B}$ are time-independent but different; consequently, constant type Il drift is present (sec Fig. 11a). However, since $\hat{A}(x), \hat{B}(x)$ are symmetric under spatial rellection, type I drift is absent. In contrast, at $\mu=4.3$ we find strongly chaotic but spatially symmetric oscillations as illustrated in Figs. $11 \mathrm{~b}, \mathrm{c}$. Since the attractor is symmetric on average about $A=B$ there is no net type II drift. Observe that at some times the large amplitude bumps coincide (i.c., the solution lies close to the invariant space $A=B$, while at other times the bumps are maximally separated, a behavior reminiscent of the states found by Bayliss and Matkowsky [22] in a combustion problem. Fig. $11 \mathrm{~b}$ suggests that this chaotic behavior is due to the presence of an unstable rellection-symmetric solution similar to that shown in Fig. 11a but lying in the $A=B$ 
a)
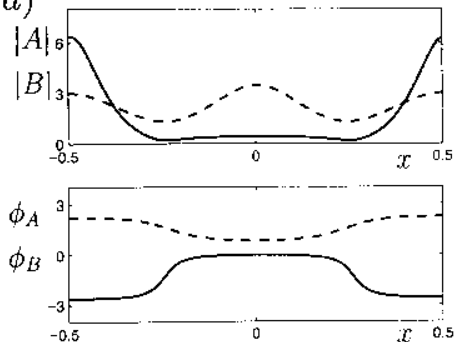

c)

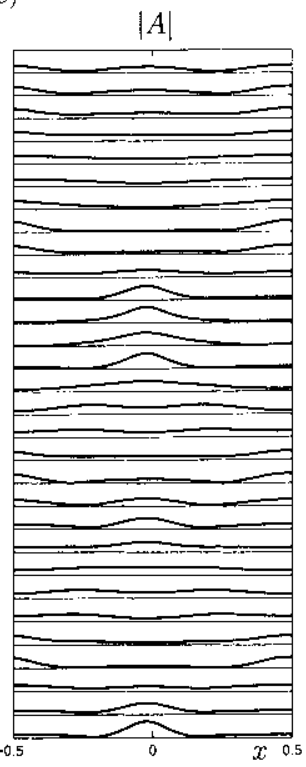

$|B|$

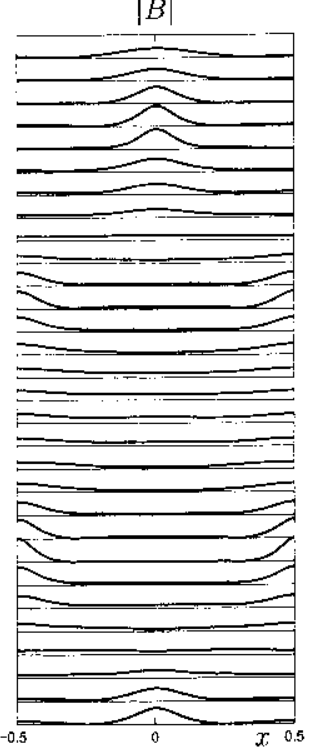

b)
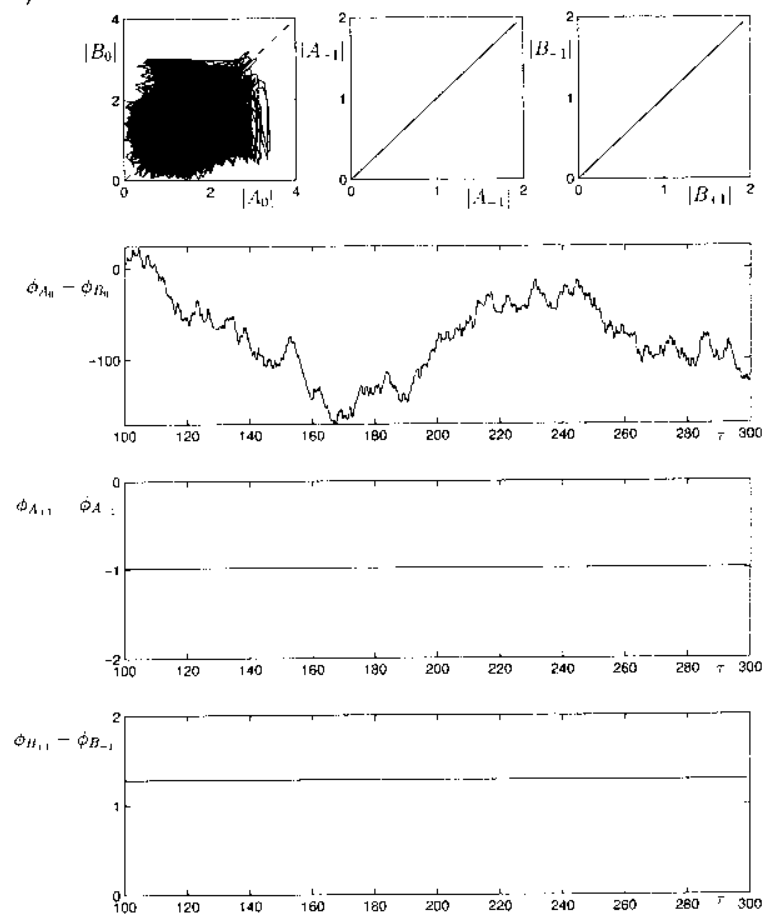

Fig. 11. As for Fig. 9 but for the parameters of Fig. $7 \mathrm{~b}$; (a) shows the amplitude profiles when $\mu=4.4$ : (b) shows the projections on the $k=0, \pm 1$ Fourier modes when $k=4.3$, showing reflection-symmetric solutions with interchange symmetry on average; (c) shows the corresponding space-time plots.

subspace. At higher values of $\mu$ (e.g., $\mu=5.5$ ) the solution is typically strongly chaotic, but with both interchange and spatial symmetries on average.

(iv) $2 \beta+1>0, \alpha<0$. In this case the uniform SW are stable for all $v>0$. As in $A$, we have found no spatially nonuniform or time-dependent attractor in this case.

\subsection{Space-time reconstruction}

We have seen that both with and without lateral walls the first nontrivial state of the system is a spatially uniform standing wave (SW). This state is characterized by time-independent amplitudes $A$ and $B$ such that $|A|=|B|=R$ (with $R$ given by (46)) and is stable near onset (because $v=0$, see Fig. 2). Subsequent instabilities generate states with nonuniform amplitudes and may preserve the equality $A=B$ (up to translations, reflection and phase shifts) or break it. The reconstructed physical field $\mathbf{u}(x, t)$ takes the form

$$
\mathbf{u}(x, t)=\left\{|A| \mathrm{e}^{\mathrm{i} L(\Omega t+k x)+i \phi_{A}}+|B| \mathrm{e}^{\mathrm{i} L(\Omega t-k x)+\mathrm{i} \phi_{B}}+\text { c.c. }\right\} \mathbf{u}_{0}+\cdots,
$$


a)

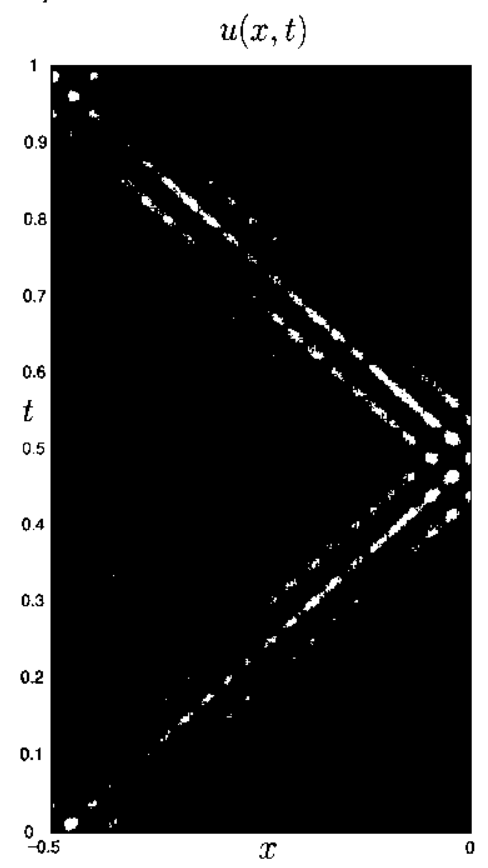

b)

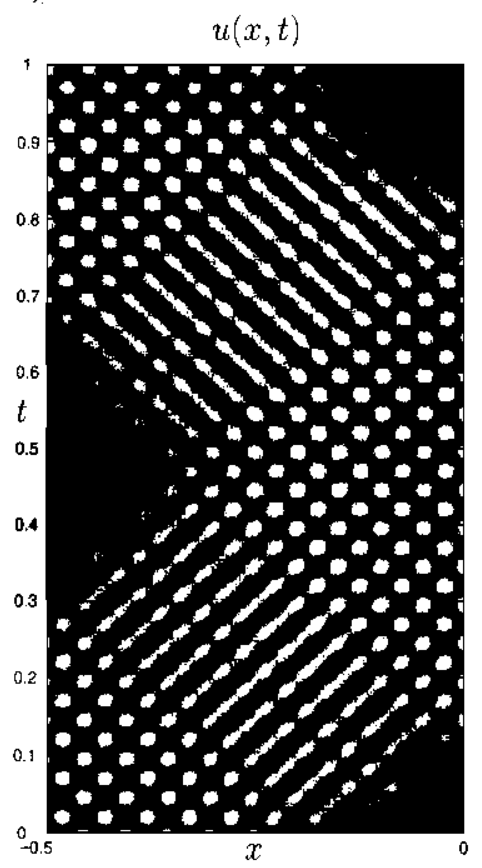

c)

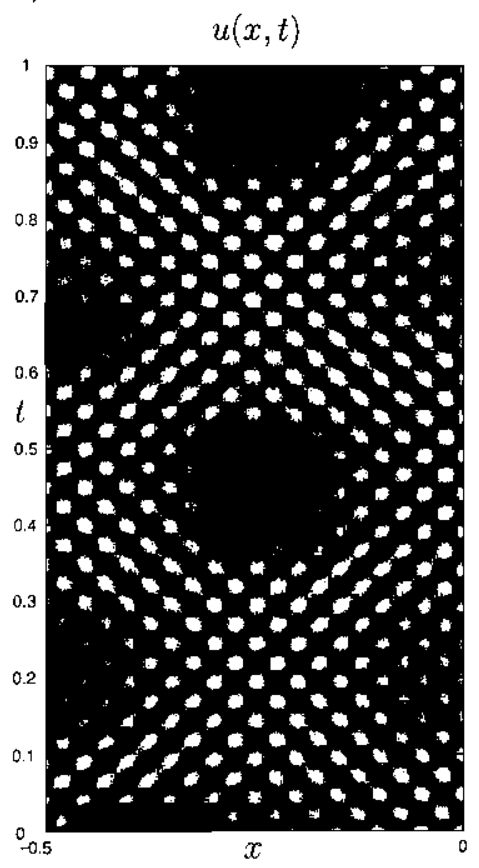

Fig. 12. The (scalar) field $u(x, t)$, reconstructed from the solution in (a) Fig. 8a, (b) Fig. 6b and (c) Fig. 4b.

where $A(\xi, \tau)=A(x-t, t / L)$ and $B(\eta, \tau)=B(x+t, t / L)$ are calculated from (38)-(40) or (41) and (42), $\phi_{A}(x-t, t / L)$ and $\phi_{B}(x+t, t / L)$ are the phases of $A$ and $B$, and $L \gg 1$. In the space-time plots we always take $k L=20 \pi$ and $\Omega=k$ eventhough the group and phase velocities are usually unequal. The space-time plots are drawn around a fixed value of the slow time $\tau=t / L$. Thus in this section we focus first and foremost on the dynamics on the advection timescale, i.e., the timescale on which spatial inhomogeneities are advected by the group velocity; the dynamics on this timescale are always periodic. Any time-dependence occurring on the slower timescale $\tau$ described in the preceding sections (e.g., phase slips) will occur too slowly to be visible in the reconstructions that follow, but manifests itself as a slow modulation of the periodic solutions on the advection timescale, as discussed at the end of this section.

We consider first the case when $A$ and $B$ remain equal, i.c., the ficlds corresponding to the various solutions of the system (41) and (42). Since in this case the solutions $C(x, \tau)$ are oblained for $-1 / 2<x<1 / 2$ the reconstructed field occupies $-1 / 2<x<0$; any inhomogeneity that is advected on the advection timescale is reflected at the latcral walls (which are now at $x=-1 / 2$ and $x=0$ ). In addition to the spatially uniform standing wave (SW), the sy stem cxhibits a varicly of propagative modes, which can be spatially symmet ric or not. The simplest nonsymmetric propagative mode arises when $C(x)$ has one localized maximum in $-1 / 2<x<1 / 2$. When this maximum lies in $-1 / 2<x<0$ a pulse of progressive waves travels to the left; when this pulse recnters the domain through $x=1 / 2$ a right-traveling pulse appears at $x=-1 / 2$. These pulses propagate in the same direction as the corresponding carrier wave. Consequently, the space-lime reconstruction on the advection timescale yiclds a periodic pattern of pulses that repeatedly "bounce" off the lateral walls, as in Fig. 12a [10,31]. This figure was generated from the single pulse profile shown in Fig. 8a. Away from the maximum the amplitudes of the left-and right-traveling components will be comparable and the reconstructed ficld will therefore consist of strongly interfering carrier waves, i.c., of (smaller amplitude) SW. These background SW are clearly visible in the reconstruction. If, instead, 
$C(x)$ has a single localized minimum one obtains a similar pattern of bouncing pulses, but the pulse now propagates in a direction opposite to that of the carrier wave and corresponds to an amplitude deficit propagating through a background of larger amplitude SW. We show an example in Fig. 12b generated from the solution shown in Fig. 6b. Symmetric propagative modes arise when $C(x)$ has a pair of localized maxima, a distance $1 / 2$ apart. Since the localized maximum in $-1 / 2<x<0$ is associated with an enhanced amplitude of left-traveling waves in $-1 / 2<x<0$ while the maximum in $0<x<1 / 2$ is associated with enhanced amplitude of right-traveling waves in $-1 / 2<x<0$ it follows that the resulting space-time reconstruction will consist of a prominent symmetric criss-cross pattern of "pulses" that propagate in the same direction as the carrier waves. We show an example of such a state in Fig. 12c reconstnicted from the symmetric $C(x)$ profile shown in Fig. $4 \mathrm{~b}$. In contrast, if the solution $C(x)$ possesses two minima instead of maxima, the reconstructed space-time evolution of the field still consists of a criss-cross pattern but now the pulses propagate in opposite direction to that of the carrier waves. When more than one localized pulse is present the distinction between bouncing and criss-cross patterns becomes less clear-cut, but depends on the distance between the pulses. When this distance is small the system exhibits a bouncing pattern in which a pair of pulses travel together from one wall to the other; if instead this distance is close to $1 / 2$ the system exhibits a criss-cross pattern. Also, the distinction between the progressive waves in the pulses and the SW background is not always clear-cut, since it depends on the quantity $\|A|-| B\| /(|\Lambda|+|B|)$. When this quantity is small the system resembles a SW, while if it is close to one it resembles a progressive wave. Since these statements apply to the amplitudes of the carrier waves a progressive wave in the above sense is only present if it is localized; it then propagates at the group velocity. The dynamics on the advection timescale just described are of course modulated on the even slower timescale $\tau$. For instance, the attractor in Fig. 4 b yields a periodic oscillation between the criss-cross pattern in Fig. $12 \mathrm{~b}$ and a SW. Note that even though these states are generated by solutions of Eqs. (38)-(40) in the invariant subspace $A=B$ these solutions do not resemble simple standing waves.

More generally, nonuniform solutions of Eqs. (38)-(40) with $A \neq B$ yield a variety of propagative modes. Again these come in two types. Suppose first that $A(x)$ and $B(x)$ have localized maxima. If $A(x)=B(x)$ to within a translation we recover the criss-cross patterns just described. However, it is possible that $A(x) \neq B(x)$ in which case the criss-cross pattern is asymmetrical. We show an example in Fig. 13a obtained from the profiles $A(x), B(x)$ shown in Fig. 1 la. Similar symmetrical or asy mmetrical criss-cross patterns arising from localized minima can also be found. More interesting are the drift modes that arise when one amplitude is constant and the other has a localized maximum or minimum. Since $\|A\|_{L_{2}}=\|B\|_{L_{2}}$, if $A$ dominates $B$ in one part of the domain $-1 / 2<x<1 / 2, B$ must dominate $A$ in the remainder. Consequently a typical drift mode consists of a pulse traveling either in the direction of the carrier wave or against it, while in the rest of the domain the amplitudes of left-and right-traveling waves are comparable, and the space-time reconstruction looks like a SW. Note that in contrast to the bouncing pulses considered above these drift modes consist of a pulse which propagates along the annulus always in the same direction. We show an cxample of such a drift mode in Fig. 13b, obtained from the solution shown in Fig. 10a.

Note that we use here the term drift to refer to advection at the group velocity. More generally, whenever the symmetry (23) is broken, we expect a drift in the reconstructed pattern (which can be secn as type I drift in the physical variable $\mathbf{u}$ ). This symmetry is broken either if (i) $A$ and/or $B$ lose spatial rellection sy mmetry, or if (ii) the sy mmetry with respect to the invariant subspace $A=B$ is lost. In case (i) the associated type I drift of $A$ and/or $B$ results in an $O(\varepsilon)$ drift velocity (in unscaled variables) so that the aclual group velocities of the counterpropagating wavetrains comprising the pattern are $1 \pm \mathcal{O}(\varepsilon)$. Thus the loss of reflection symmetry splits the effective group velocities of the constituent wavetrains, i.c., the velocities of the counterpropagating pulses of progressive waves. In contrast, in case (ii) the associated type Il drift splits the effective frequencies of the constituent waves and thus manifests itself in a slight difference between the phase speeds of the counterpropagating carriers. These drifts are too slow to be discernible in the space-lime reconstructions in Figs. 12 and 13 and can only be inferred from the associated asymmetries. In addition, they can be masked by other slow motions of the reconstructed pattern. 
a)

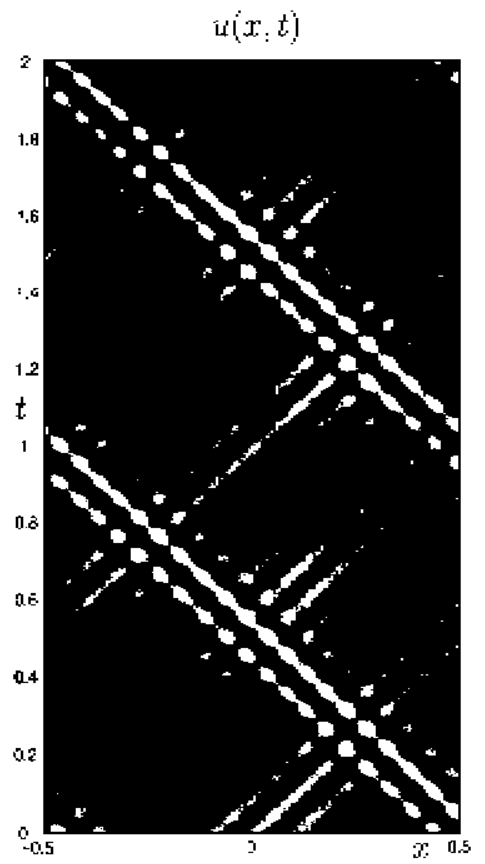

b)

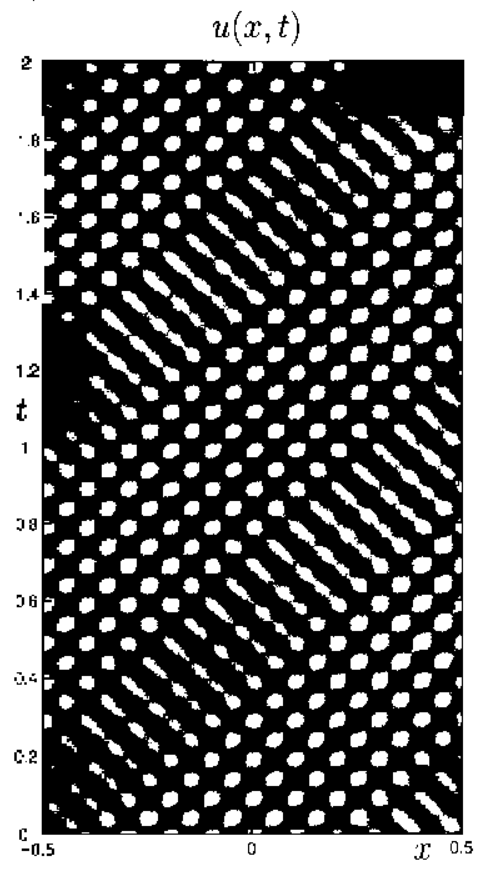

Fig. 13. The (scalar) field $u(x, t)$, reconstructed from the solution in (a) Fig. 11a and (b) Fig. 10a.

At first sight the criss-cross patterns identified above are reminiscent of the compression modes encountered experimentally in the Faraday system in annular geometry [9]. However, the motion of the nodes in the criss-cross patterns identified here is not directly associated with any compression of the wavelength. Instead, it is associated with (kinematic) phase slips in the reconstructed pattern at the boundaries of the traveling envelope pulses. These phase slips do not change the overall phase difference across the periodic domain, and differ from the ones discussed in Sections 4.1 and 4.2 which require either $A$ or $B$ to vanish.

\section{Discussion and conclusions}

In this paper we have attempted a brief survey of the possible solutions of a pair of parametrically driven weakly dissipative nonlinear Schrödinger equations with nonlocal coupling. These equations apply sufficiently close to threshold of the primary parametric instability and arise whenever the group velocity of the waves is comparable to their phase velocity. The range of validity of these equations is determined by the aspect ratio of the system, assumed to be moderately large, as described in Section 1. In all cases the primary instability is to a pattern of standing waves of uniform amplitude and phase-locked to the drive. However, the secondary instabilities of this state depend on the coefficients in these equations; we have identified four distinct regimes exhibiting different dy namical behavior arising from such an instability. For \%cro detuning these results are summarized in the bifurcation diagrams presented in Figs. 3,5 and 7. To minimi/ce the number of parameters we have not considered nonzero values of the detuning parameler, although we are aware that detuning can introduce new types of transitions not discussed in this paper. Throughout we have compared the results with periodic boundary conditions with the corresponding ones for (nearly) Neumann boundary conditions. This comparison revealed a number of differences between these two 
cases, particularly at larger forcing amplitudes, and demonstrated that with the latter boundary conditions the drift mode becomes a bouncing mode as a result of reflection from the boundaries. In addition we have emphasized the role played by reflection symmetries. Two such sy mmetries were found to be particularly important. The absence of the first symmetry, an interchange between the amplitudes $A$ and $B$ of left-and right-traveling waves, was found to be responsible for the observed type II phase drifts. Such drifts are created spontaneously at a bifurcation that breaks this interchange symmetry; their net effect is to split the overall velocities of the left-and right-traveling carrier waves. We also noted that the spontaneous breaking of a second symmetry, a symmetry under spatial reflection about a suitably chosen origin, is accompanied by a type I drift. Bifurcations of this type arise as a result of a parity-breaking instability, and can involve both stationary and time-dependent states. Their principal effect is to introduce slow large-scale drifts of the patterns. This drift splits the effective group velocities of the constituent wavetrains.

Riecke [32] has studied a related problem in which the group velocity is taken to be small, $\mathcal{O}\left(L^{-1}\right)$. In this case the resulting amplitude equations are coupled more strongly, with a local coupling between the two amplitudes and the parametric forcing. In particular, Riecke has studied, in the fully dissipative regime, the stability of the corresponding spatially uniform standing waves with respect to long wavelength perturbations and identified a bounded region in the $(k, \mu)$ plane where such waves are stable. In this system double phase slips occur at large enough forcing in which a phase slip that takes unstable standing waves inside the Eckhaus boundary is reversed some time later, leading to persistent periodic or chaotic phase slips. Under appropriate circumstances this process may result in localized spatio-temporal chaos [23,24]. In our system we have found no evidence for such localized spatio-temporal chaos, presumably because of the global coupling due to $\mathcal{O}(1)$ group velocity.

The above discussion suggests that a variety of interesting and complex dy namics is to be expected near the edges of resonance tongues in one-dimensional, moderately large aspect ratio, low viscosity Faraday systems. Consequently it would be on interest to relate the four types of basic solutions identified here (the drift and criss-cross patterns with periodic boundary conditions, and their counterparts with (near-) Neumann boundary conditions) to experimentally observed patterns such as those of Douady et al. [9] on the Faraday system in an annulus. In fact the application of the nonlocal equations to such systems is not straightforward even if the experiments satisfy the conditions for nearly inviscid dynamics. This is because of the presence of two types of mean flows that accompany the Faraday waves and interact with them. In a moderately large aspect ratio system these mean flows separate into a nearly inviscid flow and a viscous flow, of the type considered by Pierce and Knobloch [33] and Higuera et al. [34], respectively. The nearly inviscid mean flow is closely related to that described by the Davey-Stewartson equation in the large aspect ratio but strictly imviscid limit. The viscous mean flow is essentially the streaming flow that is driven at the edge of oscillatory boundary layers $[35,36]$ and applies in the low (but nonzero) viscosity limit in finite aspect ratio systems. Neither of these descriptions apply to the large aspect ratio Faraday system which typically combine the effects of both large aspect ratio and small but finite viscosity. However, under appropriate conditions (c.g., in two-dimensional containers of depth large compared to the wavelength of the primary modes) the nonlocal amplitude equations do in fact apply, with the various coefficients calculated as follows: the group velocity (which is necessary for our scaling) and dispersion are calculated from the inviscid dispersion relation; $\delta$ and $\mu$ can be taken from [37,38], and $\beta$ and $\gamma$ are taken from [33]. Since the sign of the dispersion changes between the gravity and capillary limits, and the signs of the nonlinear coefficients change when the physical parameters are varied we expect to sec, in large aspect ratio annular containers, both the drift modes and the various types of criss-cross modes identified in Section 4. However, even with mean flows included, the resulting description will not describe the Eckhaus instabilities reported by Douady et al. [9], suggesting that these experiments fall within the hyperbolic regime described in [14]. Note also that Fauve et al. [39] describe the observed drift instability of uniform standing waves as the bifurcation to a stationary solution with unequal but uniform amplitudes $A, B$. Such solutions are not present in the weakly dissipative nonlocal description given here which requires that the $\mathrm{L}^{2}$ norms 
of both amplitudes are identical. In particular the instability described by Fauve et al. requires a nonzero real part of the nonlinear cross-coupling coefficient between the two waves. We also remark that Thual et al. [40] use the term drift instability to refer to the parity-breaking instability of the basic standing wave, and not to envelope modes in which one amplitude is uniform in space and the other not. Moreover, the large-scale compression mode described by Douady et al. $[9,39]$ differs from the criss-cross modes described above, as explained at the end of Section 4.

\section{Acknowledgements}

This work was supported by The National Aeronautics and Space Administration under Grant NAG3-2152. The work of CM and JMV was partially supported by DGICYT and NATO under Grants PB97-0556 and CRG-970032.

\section{References}

[1] H. Riecke, J.D. Crawford, K. Knobloch, Time-modulated oseillatory convection, Phys. Rev. I ett. 61 (1988) 1942-1945.

[2] D. Walgraef, External forcing of spatio-temporal pattems, Europhys. Lett. 7 (1988) 485-491.

[3] J. Miles, D. Ilenderson, Parametrically forced surface waves, Ann. Rev. Fluid Mech. 22 (1990) 143-165.

[4] R.D. Pierce, E. Knobloch, Evolution equations for counterpropagating edge waves, J. Fluid Mech. 264 (1994) 137163.

[5] K. Knobloch, J.I). (ribbon, Coupled N].S equations for counterpropagating waves in systems with reflection symmetry, Phys. 1.ett. A 154 (1991) $35.3-356$.

[6] I. Rehberg, S. Rasenat, J. Fineberg, M. d la Torre Juarez, V. Steinberg, Temporal modulation of tratveling waves, Phys. Rev. Letl. 61 (1988) $2449-2452$.

[7] S.G.K. Tennakoon, C.D. Andereck, J.J. Hegsetl, H. Riecke, Temporal modulation of traveling waves in the flow between rotating cylinders with broken azimuthal symmetry, Phys. Rev. F. 54 (1996) 5053-5065.

[8] A.B. F.zerskii, M.I. Rahinovich, V.P. Reutov, I.M. Starohinets, Spatiotemporal chaos in the parametric excitation of a capillary ripple, Sov. Phys. JETP 64 (1986) 1228-1236.

[9] S. Douady, S. Fatuve, O. Thual, Oscillatory phase modulation of parametrically forced surface waves, Europhys. Lell. 10 (1989) 309-315.

[10] E. Knobloch, J. De Luca, Amplitude equations for travelling wave convection, Nonlinearity 3 (1990) 975980.

[11] C. Alvarez-Pereira, I. W. Vega, On the pulsating instability of two-dimensional flames, Fur. I. Appl. Math. 3(1992) 55-73.

[12] C. Martel, J.M. Vega, Finite size effeets near the onset of the oscillatory instability, Nonlinearity 9(1996) 1129-1171.

[13] I. Keller, A. Oron, P.Z. Barr-Yoseph, Regular and irregular regimes of binary fluid convection excited by parametric resonance, Phys. Rev. E 55 (1997) 3743-3746.

[14] C. Martel, J.M. Vega, Dynamics of a hyperbolic system that applies at the onset of the oscillatory instability, Nonlinearity 11 (1998) $105-142$.

[15] F. Knobloch, Remarks on the use and misuse of the (rinzlourg-I andau equation, in: A. Joelman, A. van Harten (Lds.), Nonlinear l)ynamics and Pattern Formation in the Nattural Environment, Longman, New York, 1995, pp. 130-146.

[16] J.D. Crawford, M. Golubitsky, M.G.M. Gomes, E. Knobloch, I.N. Stewart, Boundary conditions as symmetry constraints, in: M. Roberts, I. Stewart (Eds.) Singularity Theory and its Applications, Warwick 1989, Part II., Lecture Notes in Mathematics, vol. 1463, Springer, Berlin, 1991, pp. $63-79$.

[17] J.1). Crawford, E. Knobloch, Symmetry and symmetry-breaking bifureations in fluid meehanies, Ann. Rev. Fluid Mech. 23 (1991) $341-387$.

[18] J.M. Greene, J.-S. Kim, The steady states of the Kuramolo-Sivashinsky equation, Physica D 33 (1988) 99-120.

[19] G. Dangelmayr, J. IJettel, E. Knobloch, Parity-breaking bifurcation in inhomogeneous systems, Nonlinearity 10 (1997) $1093-1114$.

[20] A.S. Landsberg, E. Knobloch, Direction-reversing traveling waves, Phys. Lett. A 159 (1991) 1720.

[21] F. Knobloch, 1).R. Moore, A minimal model of binary fluid convection, Phys. Rev. A 42 (1990) 4693-4709.

[22] A. Bayliss, B.I. Matkowsky, Interaction of counterpropagating hot spots in solid fuel combustion, Physica 1) 128 (1999) 18-40.

[23] G.D. Granzow, II. Riecke, Phase diffusion in localized spatio-lemporal amplitude chatos, Phys. Rev. Lell. 77 (1996) $2451-2454$.

[24] G.D. Granzow, H. Riecke, Double phase slips and spatio-temporal chaos in a model for parametrically excited standing waves, SIAM J. Appl. Math. 59 (1998) 900919.

[25] J.W. Miles, Parametrically excited solitary waves, J. Fluid Mech. 148 (1984) 451-460.

[26] I. Melbourne, M. Dellnitz, M. Golubitsky, Structure of symmetric attractors, Arch. Rat. Mech. Anal. 123 (1993) 75-98

[27] P. Ashwin, J. Buescu, I. Stewart, From altractor to chaotic saddle: at tale of transverse instability, Nonlinearity 9 (1996) $703-737$.

[28] P. Ashwin, R. Covas, R. Tavakol, Transverse instability for non-1normal parameters, Conlinearity 12 (1999) 563567.

[29] M.R.F.. Proctor, J. I ega, Secondary bifurcations and symmetry breaking as a route towards spatiotemporal disorder, Int. J. Bif. Chaos 5 (1995) $841-848$. 
[30] E. Covals, R. Talvakol, P. Ashwin, A. Tworkowski, J.M. Brooke, In-out intermittency in PDE and ODE models of axisymmetric mean-field dynamos, preprint.

[31] A.E. Deane, E. Knobloch, J. Toomre, Traveling waves in large-aspect-ratio thermosolutal convection, Plyss. Rev. $\Lambda 37(1988) 18171820$.

[32] H. Riecke, Stable wave-number kinks in parametrically exeited standing waves, Furophys. 1.ett. 11 (199(J) 213-218.

[33] K.1). Pieree, F.. Knobloch, (On the modulational stability of traveling and standing waves, Phys. Fluids 6 (1994) 1177-1 190.

[34] M. Iliguera, J.A. Nicolas, J.M. Vega, Coupled amplitude-streaming flow equations for the evolution of counter-rotating, nearly-inviscid surface waves in finite axisymmetric geometries, preprint.

[35] H. Schlichting, Berechnung ebener periodischer Grenzschichtströmungen, Z. Pliys. 33 (1932) 327335.

[36] M.S. Longuet-Higgins, Mass transport by water waves, Phil. Trans. R. Soc. $\Lambda 245$ (1953) 535581.

[37] C. Martel, E. Knobloch, l)amping of nearly inviscid water waves, Phys. Rev. F, 56 (1997) 5544-5548;

[38] 'T: Kakutani, K. Matsuuchi, Fffect of viscosity on long gravity waves, J. Phys. Soc. Japan 39 (1975) 2.37-246.

[39] S. Fauve, S. Douady, O. Thual, Drift instabilities of cellular pattems, J. Phys. II 1 (1991) 311-322.

[40] O. Thual, S. Douady, S. Fauve, Parametric instabilities, in: E. Tirapegui, D. Villaroel (Eds.), Instibilities and Aonequilibrium Structures II, Kluwer Academic Publishers, Dordrecht, 1989, pp. 227237. 